\title{
Compact numerical solutions to the two-dimensional repulsive Hubbard model obtained via nonunitary similarity transformations
}

\author{
Werner Dobrautz ${ }^{*}$ and Hongjun Luo ${ }^{\dagger}$ \\ Max Planck Institute for Solid State Research, Heisenbergstraße 1, 70569 Stuttgart, Germany \\ Ali Alavi \\ Max Planck Institute for Solid State Research, Heisenbergstraße 1, 70569 Stuttgart, Germany \\ and Department of Chemistry, University of Cambridge, Lensfield Road, Cambridge CB2 1EW, United Kingdom
}

(Received 9 November 2018; published 8 February 2019)

\begin{abstract}
Similarity transformation of the Hubbard Hamiltonian using a Gutzwiller correlator leads to a non-Hermitian effective Hamiltonian, which can be expressed exactly in momentum-space representation and contains threebody interactions. We apply this methodology to study the two-dimensional Hubbard model with repulsive interactions near half filling in the intermediate interaction strength regime $(U / t=4)$. We show that at optimal or near optimal strength of the Gutzwiller correlator, the similarity-transformed Hamiltonian has extremely compact right eigenvectors, which can be sampled to high accuracy using the full configuration interaction quantum Monte Carlo (FCIQMC) method and its initiator approximation. Near-optimal correlators can be obtained using a simple projective equation, thus obviating the need for a numerical optimization of the correlator. The FCIQMC method, as a projective technique, is well suited for such non-Hermitian problems, and its stochastic nature can handle the three-body interactions exactly without undue increase in computational cost. The highly compact nature of the right eigenvectors means that the initiator approximation in FCIQMC is not severe and that large lattices can be simulated, well beyond the reach of the method applied to the original Hubbard Hamiltonian. Results are provided in lattice sizes up to 50 sites and compared to auxiliary-field QMC. New benchmark results are provided in the off-half-filling regime, with no severe sign problem being encountered. In addition, we show that methodology can be used to calculate excited states of the Hubbard model and lay the groundwork for the calculation of observables other than the energy.
\end{abstract}

DOI: 10.1103/PhysRevB.99.075119

\section{INTRODUCTION}

The fermionic two-dimensional Hubbard model [1-3] with repulsive interactions is a minimal model of itinerant strongly correlated electrons that is believed to exhibit extraordinarily rich physical behavior. Especially in the past thirty years, it has been intensively studied as a model to understand the physics of high-temperature superconductivity observed in layered cuprates [4]. Its phase diagram as a function of temperature, interaction strength, and filling includes antiferromagnetism, the Mott metal-insulator transition, unconventional superconductivity [5] with $d$-wave pairing off half filling, striped phases, a pseudogap regime, and charge and spin density waves [6]. Confronted with such a plethora of physical phenomena, accurate numerical results are indispensable in resolving various competing theoretical scenarios.

\footnotetext{
*w.dobrautz@fkf.mpg.de

†h.luo@fkf.mpg.de

${ }^{\ddagger}$ https://www.fkf.mpg.de/alavi; a.alavi@fkf.mpg.de
}

Published by the American Physical Society under the terms of the Creative Commons Attribution 4.0 International license. Further distribution of this work must maintain attribution to the author(s) and the published article's title, journal citation, and DOI.
Unfortunately the numerical study of the 2D Hubbard model has proven extraordinarily challenging, particularly in the off-half-filling regime with intermediate-to-strong interaction strengths $U / t=4-12$. Major difficulties include severe sign problems for quantum Monte Carlo (QMC) methods, while the 2D nature of the problem causes convergence difficulties for density matrix renormalization group (DMRG) [7-9] based methodologies which have otherwise proven extremely powerful in 1D systems. Nevertheless extensive numerical studies have been performed with a variety of methods, such as variational [10-14], fixed-node [15-17], constrained-path auxiliary field [18-20], determinantal [21] and diagrammatic [22] QMC, dynamical [23-25] and variational $[26,27]$ cluster approximations (DCA/VCA), dynamical mean-field theory (DMFT) [28-32], density matrix renormalization group (DMRG), and variational tensor network states $[33,34]$. Thermodynamic limit extrapolations have been carried out with the aim of assessing the accuracy of the methodologies in various regimes of interaction, filling factor, and temperature [35-37]. On the other hand each of these methods incurs systematic errors which are extremely difficult to quantify and there is an urgent need to develop methods in which convergence behavior can be quantified internally.

In this paper, rather than attempting a direct numerical attack on the 2D Hubbard Hamiltonian with a given technique, we ask whether there is an alternative exact reformulation of 
the problem, the solution of which is easier to approximate than that of the original problem. If this is the case (and this is obviously highly desirable), it should be demonstrable within the framework of a given technique, without reference to any other method. The physical basis for any observed simplification should be transparent. Such an approach turns out to be possible, at least for intermediate interaction strengths, based on a Gutzwiller nonunitary similarity transformation of the Hubbard Hamiltonian.

The Gutzwiller ansatz [2,38] and Gutzwiller approximation [39-43] are intensively studied methods to solve the Hubbard model. The parameter of the ansatz is usually optimized to minimize the energy expectation value by variational Monte Carlo schemes [44,45] based on a single Fermi-sea reference state. It has been long realized that the simple Gutzwiller ansatz misses important correlations [46-48], especially in the strong-interaction regime of the Hubbard model. More general, Jastrow-like [49] correlators, including density-density [50] and holon-doublon [51,52], have been proposed to capture more physical features within the ansatz. In addition the Fermi-sea reference function has been extended to Hartree-Fock (HF) spin density waves [53] and BCS [54] wave functions [11,55-62] including antiferromagnetic [14,63] and charge order [64]. Recently there have been developments of a more efficient diagrammatic expansion of the Gutzwiller wave function (DE-GWF) [61,65], extensions of the Gutzwiller approximation to quasiparticle excitations [66] and linear response quantities [67], and the combination with the Schrieffer-Wolff transformation [68] to capture Mott physics beyond the Brinkman-Rice scenario.

An alternative strategy is to use a Gutzwiller correlator to perform a nonunitary similarity transformation of the Hubbard Hamiltonian, whose solution can be well approximated using a Slater determinant. Such an approach is reminiscent of the quantum chemical transcorrelated method of Boys and Handy [69,70], as well as Hirschfelder [71], in which a nonHermitian Hamiltonian is derived on the basis of a Jastrow factorization of the wave function.

This idea was applied by Tsuneyuki [72] to the Hubbard model by minimizing the variance of the energy based on projection on the HF determinant. Scuseria and coworkers [73] and Chan et al. [74] have recently generalized to general two-body correlators and more sophisticated reference states, where the correlator optimization was not performed in a stochastic variational Monte Carlo manner, but in the spirit of coupled-cluster theory, by projecting the transformed Hamiltonian in the important subspace spanned by the correlators.

These methods have in common that they are based on a single reference optimization of the correlation parameters and thus the energy obtained is on a mean-field level. We instead would like to fully solve the similarity-transformed Hamiltonian in a complete momentum-space basis. We will use a single reference optimization, based on projection $[73,74]$, to generate a similarity-transformed Hamiltonian (non-Hermitian with 3-body interactions), whose ground-state solution (right eigenvector) will be using the projective full configuration interaction quantum Monte Carlo (FCIQMC) [75] method.

The remainder of the paper is organized as follows: In Sec. II we recap the derivation of the Gutzwiller similarity-transformed Hubbard Hamiltonian and the projective solution based on the restricted Hartree-Fock determinant. We also present analytic and exact diagonalization results, to illustrate the influence of the transformation on the energies and eigenvectors. In Sec. III we recap the basics of the FCIQMC method and necessary adaptations for its application to the similarity-transformed Hubbard Hamiltonian in a momentum-space basis, named similarity-transformed FCIQMC (ST-FCIQMC). In Sec. IV we benchmark the STFCIQMC method for the exact diagonalizable 18-site Hubbard model and present ground- and excited-state energies. We observe an increased compactness of the right eigenvector of the non-Hermitian transformed Hamiltonian. We also compare the results obtained with our method for nontrivial 36- and 50-site lattices, at and off half filling, with interaction strengths up to $U / t=4$. In Sec. $\mathrm{V}$ we conclude our findings and explain future applications for observables other than the energy and correct calculation of left and right excited-state eigenvectors.

\section{THE SIMILARITY-TRANSFORMED HAMILTONIAN}

We would like to solve for the ground-state energy of the two-dimensional, single-band Hubbard model [1-3] with the Hamiltonian in a real-space basis,

$$
\hat{H}=-t \sum_{\langle i j\rangle, \sigma} a_{i, \sigma}^{\dagger} a_{j, \sigma}+U \sum_{l} n_{l, \uparrow} n_{l, \downarrow},
$$

$a_{i, \sigma}^{(\dagger)}$ being the fermionic annihilation (creation) operator for site $i$ and spin $\sigma, n_{l, \sigma}$ the number operator, $t$ the nearestneighbor hopping amplitude, and $U \geqslant 0$ the on-site Coulomb repulsion. We employ a Gutzwiller-type ansatz [2,40,76] for the ground-state wave function

$$
\begin{aligned}
|\Psi\rangle & =g^{\hat{D}}|\Phi\rangle=e^{\hat{\tau}}|\Phi\rangle, \quad \text { with } \\
\hat{\tau} & =\ln g \hat{D}=J \sum_{l} n_{l, \uparrow} n_{l, \downarrow} \quad \text { and } \quad 0 \leqslant g \leqslant 1,
\end{aligned}
$$

where $\hat{D}$ is the sum of all double occupancies in $|\Phi\rangle$, which are repressed with $0 \leqslant g \leqslant 1 \rightarrow-\infty<J \leqslant 0$.

In the Gutzwiller ansatz, $|\Phi\rangle$ is usually chosen to be a single-particle product wave function $[2,77],\left|\Phi_{0}\right\rangle$, such as the Fermi-sea solution of the noninteracting $U=0$ system, or other similar forms such as unrestricted Hartree-Fock spin density waves [53] or superconducting BCS wave functions [55]. The parameter $J$ is usually optimized via variational Monte Carlo (VMC) [42], minimizing the expectation value

$$
E_{\mathrm{VMC}}=\min _{J} \frac{\left\langle\Phi_{0}\left|e^{\hat{\tau}} \hat{H} e^{\hat{\tau}}\right| \Phi_{0}\right\rangle}{\left\langle\Phi_{0}\left|e^{2 \hat{\tau}}\right| \Phi_{0}\right\rangle}
$$

In this work, however, $|\Phi\rangle$ is taken to be a full configuration interaction (CI) expansion in terms of Slater determinants

$$
|\Phi\rangle=\sum_{i} c_{i}\left|D_{i}\right\rangle
$$


with which we aim to solve an equivalent exact eigenvalue equation

$$
\begin{aligned}
e^{-\hat{\imath} \hat{H}} e^{\hat{\imath}}|\Phi\rangle & =\bar{H}|\Phi\rangle=E|\Phi\rangle, \quad \text { with } \\
\bar{H} & =-t \sum_{\langle i j\rangle, \sigma} e^{-\hat{\tau}} a_{i, \sigma}^{\dagger} a_{j, \sigma} e^{\hat{\imath}}+U \sum_{l} n_{l, \uparrow} n_{l, \downarrow} ;
\end{aligned}
$$

$\bar{H}$ denotes a similarity-transformed Hamiltonian. Equation (6) is obtained by substituting Eq. (2) as an eigenfunction ansatz into Eq. (1) and multiplying with $e^{-\hat{\imath}}$ from the left, and due to $\left[n_{i, \sigma}, \hat{\tau}\right]=0$. The similarity transformation of Eq. (1) moves the complexity of the correlated ansatz for the wave function $|\Psi\rangle$ into the Hamiltonian, without changing its spectrum. It is a nonunitary transformation, and the resulting Hamiltonian is not Hermitian. Such similarity transformations have been introduced in quantum chemistry $[69,71,78]$ in the context of a Slater-Jastrow ansatz, were it is known as the transcorrelated method of Boys and Handy. It was first applied to the Hubbard model by Tsuneyuki [72]. The transcorrelated method has been quite widely applied in combination with explicitly correlated methods in quantum chemistry [79-82], with approximations being employed to terminate the commutator series arising from the evaluation of $e^{-\hat{\imath}} \hat{H} e^{\hat{\imath}}[83,84]$. The explicit similarity transformation of the Hubbard Hamiltonian (1) with a Gutzwiller $(2)[72,85]$ or more general correlator $[73,74]$, which can be obtained without approximations due to a terminating commutator series, has been solved on a mean-field level [72]. In the present work, we will not restrict ourselves to a mean-field solution, but solve for the exact ground state of $\bar{H}$ with the FCIQMC method $[75,86]$.

\section{A. Recap of the derivation of $\overline{\boldsymbol{H}}$}

Tsuneyuki [72] and Scuseria et al. [73] have provided a derivation of the similarity-transformed Hubbard Hamiltonian, based on the Gutzwiller and more general two-body correlators, respectively. Their derivations result in a Hamiltonian expressed in real space. Here we go one step further and obtain an exact momentum-space representation of the similarity-transformed Hamiltonian, which is advantageous in the numerical study of the intermediate correlation regime. In this representation, the total momentum is an exact quantum number, resulting in a block-diagonalized Hamiltonian. This is computationally useful in projective schemes, especially where there are near degeneracies in the exact spectrum close to the ground state, which can lead to very long projection times and be problematic to resolve. Additionally, it turns out that even in the intermediate-strength regime, the ground-state right eigenvector is dominated by a single Fermi determinant for the half-filled system. This is in stark contrast with the ground-state eigenvector of the original Hubbard Hamiltonian, which is highly multiconfigurational in this regime.

As seen in Eq. (7) we need to compute the following transformation,

$$
\hat{F}(x)=e^{-x \hat{\imath}} a_{i, \sigma}^{\dagger} a_{j, \sigma} e^{x \hat{\imath}},
$$

which can be done by introducing a formal variable $x$ and performing a Taylor expansion (cf. the BakerCampbell-Hausdorff expansion). The derivatives of (8) can be calculated as

$$
\begin{aligned}
\hat{F}^{\prime}(0) & =\left[a_{i, \sigma}^{\dagger} a_{j, \sigma}, \hat{\tau}\right]=J \sum_{l}\left[a_{i, \sigma}^{\dagger} a_{j, \sigma}, a_{l, \sigma}^{\dagger} a_{l, \sigma}\right] n_{l, \bar{\sigma}} \\
& =J a_{i, \sigma}^{\dagger} a_{j, \sigma}\left(n_{j, \bar{\sigma}}-n_{i, \bar{\sigma}}\right), \\
\hat{F}^{\prime \prime}(0) & =\left[\left[a_{i, \sigma}^{\dagger} a_{j, \sigma}, \hat{\tau}\right], \hat{\tau}\right]=J\left[a_{i, \sigma}^{\dagger} a_{j, \sigma}\left(n_{j, \bar{\sigma}}-n_{i, \bar{\sigma}}\right), \hat{\tau}\right] \\
& =J^{2} a_{i, \sigma} a_{j, \sigma}\left(n_{j, \bar{\sigma}}-n_{i, \bar{\sigma}}\right)^{2}, \\
\hat{F}^{(n)}(0) & =\left[\left[a_{i, \sigma}^{\dagger} a_{j, \sigma}, \hat{\tau}\right], \ldots, \hat{\tau}\right] \\
& =J^{n} a_{i, \sigma} a_{j, \sigma}\left(n_{j, \bar{\sigma}}-n_{i, \bar{\sigma}}\right)^{n} .
\end{aligned}
$$

With this closed form (9) the Taylor expansion can be summed up as $\hat{F}(1)=a_{i, \sigma}^{\dagger} a_{j, \sigma} e^{J\left(n_{j, \bar{\sigma}}-n_{i, \bar{\sigma}}\right)}$ and Eq. (6) takes the final form of $[72,73,85]$

$$
\bar{H}=-t \sum_{\langle i j\rangle, \sigma} a_{i, \sigma}^{\dagger} a_{j, \sigma} e^{J\left(n_{j, \bar{\sigma}}-n_{i, \bar{\sigma}}\right)}+U \sum_{l} n_{l, \uparrow} n_{l, \downarrow} .
$$

Due to the idempotency of the (fermionic) number operators, $n_{i, \sigma}^{2}=n_{i, \sigma}$, we have for $m \geqslant 1$

$$
\begin{aligned}
\left(n_{j, \sigma}-n_{i, \sigma}\right)^{2 m-1} & =n_{j, \sigma}-n_{i, \sigma}, \quad \text { and } \\
\left(n_{j, \sigma}-n_{i, \sigma}\right)^{2 m} & =n_{j, \sigma}+n_{i, \sigma}-2 n_{i, \sigma} n_{j, \sigma} .
\end{aligned}
$$

With Eq. (11) the exponential factor in Eq. (10) can be calculated as

$$
\begin{aligned}
e^{J\left(n_{i, \sigma}-n_{j, \sigma}\right)}= & 1+\sum_{m=1}^{\infty} \frac{J^{2 m-1}}{(2 m-1) !}\left(n_{j, \sigma}-n_{i, \sigma}\right) \\
& +\sum_{m=1}^{\infty} \frac{J^{2 m}}{(2 m) !}\left(n_{j, \sigma}+n_{i, \sigma}-2 n_{i, \sigma} n_{j, \sigma}\right) \\
= & 1+\sinh (J)\left(n_{j, \sigma}-n_{i, \sigma}\right)+[\cosh (J)-1] \\
& \times\left(n_{j, \sigma}+n_{i, \sigma}-2 n_{i, \sigma} n_{j, \sigma}\right) \\
= & 1+\left(e^{J}-1\right) n_{j, \sigma}+\left(e^{-J}-1\right) n_{i, \sigma} \\
& -2[\cosh (J)-1] n_{i, \sigma} n_{j, \sigma} .
\end{aligned}
$$

With Eq. (12) we can write the final similarity-transformed Hamiltonian as

$$
\begin{aligned}
\bar{H}= & \hat{H}-t \sum_{\langle i j\rangle, \sigma} a_{i, \sigma}^{\dagger} a_{j, \sigma}\left\{\left(e^{J}-1\right) n_{j, \bar{\sigma}}+\left(e^{-J}-1\right) n_{i, \bar{\sigma}}\right. \\
& \left.-2[\cosh (J)-1] n_{i, \bar{\sigma}} n_{j, \bar{\sigma}}\right\} .
\end{aligned}
$$

Formulated in a real-space basis the additional factor in Eq. (13) is simply a nearest-neighbor density-dependent renormalization of the hopping amplitude. For large interaction $U / t \gg 1$, as already pointed out by Fulde et al. [46], the simple ansatz (2) shows the incorrect asymptotic energy behavior, $E \sim-t^{2} /(U \ln U)$ instead of $E \sim-t^{2} / U$ [47,48], proportional to the magnetic coupling of the Heisenberg model for $U / t \gg 1$, due to the missing correlation between nearest-neighbor doubly occupied and empty sites. The Gutzwiller ansatz does however provide a good energy estimate in the low to intermediate $U / t$ regime. For these values of $U / t$ the momentum-space formulation is a better suited choice of basis, due to a dominant Fermi-sea determinant and thus a single reference character of the ground-state wave 
function. Thus we transform Eq. (13) with

$$
\begin{gathered}
a_{\mathbf{l}, \sigma}^{\dagger}=\frac{1}{\sqrt{M}} \sum_{\mathbf{k}} e^{-i \mathbf{k} \mathbf{l}} c_{\mathbf{k}, \sigma}^{\dagger}, \quad \begin{array}{l}
\text { into a momentum-space representations } \\
\text { become }
\end{array} \\
\sum_{\langle i j\rangle, \sigma} a_{i, \sigma}^{\dagger} a_{j, \sigma} n_{j, \bar{\sigma}}=\frac{1}{M} \sum_{\mathbf{p q k}, \sigma} \epsilon_{\mathbf{p}-\mathbf{k}} c_{\mathbf{p}-\mathbf{k}, \sigma}^{\dagger} c_{\mathbf{q}+\mathbf{k}, \bar{\sigma}}^{\dagger} c_{\mathbf{q}, \sigma} c_{\mathbf{p}, \sigma}, \\
\sum_{\langle i j\rangle, \sigma} a_{i, \sigma}^{\dagger} a_{j, \sigma} n_{i, \bar{\sigma}}=\frac{1}{M} \sum_{\mathbf{p q k}, \sigma} \epsilon_{\mathbf{p}} c_{\mathbf{p}-\mathbf{k}, \sigma}^{\dagger} c_{\mathbf{q}+\mathbf{k}, \bar{\sigma}}^{\dagger} c_{\mathbf{q}, \bar{\sigma}} c_{\mathbf{p}, \sigma}, \\
\sum_{\langle i j\rangle, \sigma} a_{i, \sigma}^{\dagger} a_{j, \sigma} n_{i, \bar{\sigma}} n_{j, \bar{\sigma}}=\frac{1}{M^{2}} \sum_{\mathbf{p q s k k ^ { \prime } , \sigma}} \epsilon_{\mathbf{p}-\mathbf{k}+\mathbf{k}^{\prime}} c_{\mathbf{p}-\mathbf{k}, \sigma}^{\dagger} c_{\mathbf{q}+\mathbf{k}^{\prime}, \bar{\sigma}}^{\dagger} c_{\mathbf{s}+\mathbf{k}-\mathbf{k}^{\prime}, \bar{\sigma}}^{\dagger} c_{\mathbf{s}, \bar{\sigma}} c_{\mathbf{q}, \bar{\sigma}} c_{\mathbf{p}, \sigma},
\end{gathered}
$$

with $\epsilon_{\mathbf{k}}$ being the dispersion relation of the lattice. The original Hubbard Hamiltonian in $\mathbf{k}$ space is

$$
\hat{H}=-t \sum_{\mathbf{k}, \sigma} \epsilon_{\mathbf{k}} n_{\mathbf{k}, \sigma}+\frac{U}{2 M} \sum_{\mathbf{p q k}, \sigma} c_{\mathbf{p}-\mathbf{k}, \sigma}^{\dagger} c_{\mathbf{q}+\mathbf{k}, \bar{\sigma}}^{\dagger} c_{\mathbf{q}, \bar{\sigma}} c_{\mathbf{p}, \sigma},
$$

while the similarity-transformed Hamiltonian in $\mathbf{k}$ space is a function of the correlation parameter $J$,

$$
\begin{aligned}
\bar{H}(J)=- & t \sum_{\mathbf{k}, \sigma} \epsilon_{\mathbf{k}} n_{\mathbf{k}, \sigma}+\frac{1}{M} \sum_{\mathbf{p q k}, \sigma} \omega(J, \mathbf{p}, \mathbf{k}) c_{\mathbf{p}-\mathbf{k}, \sigma}^{\dagger} c_{\mathbf{q}+\mathbf{k}, \bar{\sigma}}^{\dagger} c_{\mathbf{q}, \bar{\sigma}} c_{\mathbf{p}, \sigma} \\
+ & 2 t \frac{\cosh (J)-1}{M^{2}} \sum_{\mathbf{p q s k k ^ { \prime } , \sigma}} \epsilon_{\mathbf{p}-\mathbf{k}+\mathbf{k}^{\prime}} c_{\mathbf{p}-\mathbf{k}, \sigma}^{\dagger} c_{\mathbf{q}+\mathbf{k}^{\prime}, \bar{\sigma}}^{\dagger} c_{\mathbf{s}+\mathbf{k}-\mathbf{k}^{\prime}, \bar{\sigma}}^{\dagger} c_{\mathbf{s}, \bar{\sigma}} c_{\mathbf{q}, \bar{\sigma}} c_{\mathbf{p}, \sigma} \\
& \omega(J, \mathbf{p}, \mathbf{k})=\frac{U}{2}-t\left[\left(e^{J}-1\right) \epsilon_{\mathbf{p}-\mathbf{k}}+\left(e^{-J}-1\right) \epsilon_{\mathbf{p}}\right] .
\end{aligned}
$$

Compared to the original Hubbard Hamiltonian in $\mathbf{k}$ space (18), $\bar{H}$ (19) has a modified 2-body term and contains an additional 3-body interaction, which for $\mathbf{k}=0$ gives rise to parallel-spin double excitations. These are not present in the original Hamiltonian. As mentioned above, in contrast to other explicitly correlated approaches [87] this is an exact similarity transformation of the original Hamiltonian and does not depend on any approximations. Hence the spectrum of this Hamiltonian is the same as that of (1). Unlike the canonical transcorrelation ansatz of Yanai and Shiozaki [79] which employs a unitary similarity transformation, the resulting Hamiltonian (19) is not Hermitian (the non-Hermiticity arising in the two-body terms), and hence its spectrum is not bounded from below. Variational minimization approaches are not applicable. The left and right eigenvectors differ, and form a biorthogonal basis $\left\langle\Psi_{i}^{L} \mid \Psi_{j}^{R}\right\rangle=0$ for $i \neq j$. Tsuneyuki circumvented the lack of a lower bound of $\bar{H}$ by instead minimizing the variance of $\bar{H}$,

$$
\min \left\langle\Phi_{H F}\left|[\bar{H}-\langle\bar{H}\rangle]^{2}\right| \Phi_{H F}\right\rangle,
$$

which is zero for the exact wave function and positive otherwise, to determine the optimal $J_{\mathrm{var}}$.

Projective methods such as the power method [88], or a stochastic variant such as FCIQMC [86], can converge the right/left eigenvectors by multiple application of a suitable propagator, without recourse to a variational optimization procedure, and this is the technique we shall use here. Since the matrix elements of (19) can be calculated analytically and on-the-fly, the additional cost of the 3-body term is no hindrance in our calculations and we do not need to apply additional approximations, unlike other explicitly correlated approaches $[89,90]$. While complicating the calculation of observables other than the energy, due to the need to have both the left and right eigenvector of the now non-Hermitian Hamiltonian (19), the difference between the left and right eigenvectors actually proves to be beneficial for the sampling of the ground-state wave function in the FCIQMC method. This will be numerically demonstrated below in Sec. IIC. As a side note, the use of more elaborate correlators, such as density-density [50] or holon-doublon [51,52,91], is no hindrance in the real-space formulation of the Hubbard model and is currently being investigated [92], but in the momentumspace basis would lead to even higher order interactions and have not been further explored.

\section{B. Analytic results for the Hubbard model}

As a starting point we optimize the strength of the correlation factor, controlled by the single parameter $J$ from the ansatz (2), by projecting the single determinant eigenvalue equation $[\bar{H}(J)-E]\left|\Phi_{H F}\right\rangle=0$ to the single basis of the correlation factor $[73,74,85]$

$$
\left\langle(\hat{\tau}-\langle\hat{\tau}\rangle)^{\dagger} \bar{H}\right\rangle_{H F}=\left\langle\hat{\tau}^{\dagger} \bar{H}\right\rangle_{c}=0,
$$

where $\langle\ldots\rangle_{c}$ denotes a cumulant expression, where only linked diagrams are evaluated. HF denotes the state with all orbitals with $|\mathbf{k}| \leqslant k_{F}$ being doubly occupied and $k_{F}$ being the Fermi surface. Equation (22) is similar to a coupled-cluster equation. We simply report the results here [further information on the solution of Eq. (22) can be found in Appendix A]. For an infinite system at half filling, and only considering the 
TABLE I. Ground-state energy per site for the half-filled $16 \times 16$ square lattice with periodic (PBC) and mixed (anti)periodic (ABPC) boundary conditions along the $(y) x$ axis. Thermodynamic limit extrapolations (TDL) for various values of $U / t$ obtained with AFQMC [36] are denoted as $E_{\mathrm{Ref}}^{(\mathrm{TDL})}$. Results obtained by evaluating Eq. (22) and Eqs. (23) and (24) are noted as $E_{J}^{(\mathrm{TDL})}$. The optimal value of $J$ is also shown. All energies are in units of $t$.

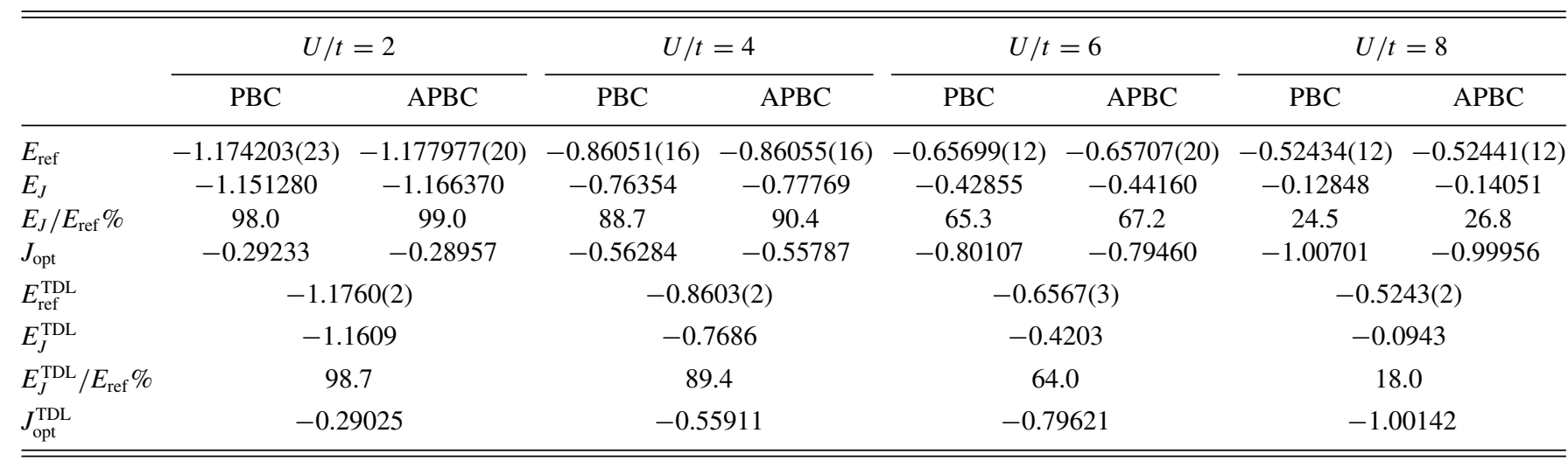

two-body contribution of Eq. (22), we can express the optimal $J$ which fulfils Eq. (22), and the corresponding total energy per site, as (see Appendix B)

$$
\begin{gathered}
J_{\mathrm{opt}}^{\mathrm{TDL}}=\sinh ^{-1}\left(-\frac{5 U \pi^{6}}{288 t\left(16+\pi^{4}\right)}\right), \\
E_{J}^{\mathrm{TDL}}=-t \frac{64}{4 \pi^{2}}+\frac{U}{4}-t J_{\mathrm{opt}}^{\mathrm{TDL}}\left(\frac{16}{4 \pi^{2}}+\frac{64}{\pi^{6}}\right) .
\end{gathered}
$$

The results of Eqs. (22)-(24) compared with auxiliary-field QMC (AFQMC) results [36] on a $16 \times 16$ half-filled square lattice are shown in Table I, for various values of $U / t$. The superscript (TDL) denotes thermodynamic limit results from Eqs. (23) and (24) for both the energy and $J$ parameter, and an absent superscript refers to the solution of Eq. (22) for the actual finite lattices. At half filling AFQMC does not suffer from a sign problem [93] and is numerically exact. One can see that the results obtained from Eqs. (22)-(24) capture most of the correlation energy for low $U / t$ as expected. For larger $U / t$, due to the missing correlation between empty and doubly occupied sites in the ansatz (2), the energies progressively deteriorate compared to the reference results. The optimal value $J_{\text {opt }}$ is also displayed in Table I. We use these values of $J$ obtained by solving Eq. (22) as a starting point for our FCIQMC calculations to capture the remaining missing correlation energy.

To compare this most basic combination of an on-site Gutzwiller correlator and a single restricted Hartree-Fock determinant as a reference in Eq. (22) we show in Table II the percentage of the energy obtained with this method to more elaborate correlators and reference states, for different system sizes $M$, numbers of electrons $n_{e l}$, and interaction strengths $U / t$. $E_{(S) U G S T}$ in Table II denotes an on-site Gutzwiller correlator with a (symmetry-projected) unrestricted Hartree-Fock reference state [85]. At half filling and $U / t \leqslant 4$ we can capture more than $80 \%$ of the energy obtained with a more elaborate reference determinant. Off half filling the recovered energy is above $90 \%$ up to $U / t=4$. For a more dilute filling of $\langle n\rangle=0.8$, for $M=100$ and $U / t=2$, the energies agree to better than $99 \%$. Although the absolute error in energy increases off half filling, as already mentioned in Refs. [73,85] the relative error actually decreases $[11,44,94]$, as can be seen in the comparison with the AFQMC reference results $[35,36,73,95], E_{\text {ref }}$ in Table II. As expected, for $U / t>4$ the results from Eq. (22) drastically deteriorate compared to $E_{(S) U G S T}$.

$E_{R / U J}$ in Table II refer to energies obtained with restricted/unrestricted Hartree-Fock reference states with a general two-body correlator [73], which includes all possible density-density correlations in addition to the on-site Gutzwiller factor. The comparison with $E_{R J}$ shows that, as already found in Ref. [73], the Gutzwiller factor is by far the most important term in a general two-body correlator for low to intermediate values of $U / t \leqslant 4$, with an agreement of over

TABLE II. Fraction of the total energy obtained with the Gutzwiller ansatz (2) based on the Hartree-Fock determinant (22) compared with a Gutzwiller correlator with an unrestricted HartreeFock reference $E_{\mathrm{UGST}}$ and subsequent symmetry projection $E_{\mathrm{SUGST}}$ [85] and a general two-body correlator with a Hartree-Fock reference $E_{R J}$ and unrestricted Hartree-Fock reference $E_{U J}$ [73] and numerically exact AFQMC reference results $[35,36,73,95]$ for different numbers of sites $M$, numbers of electrons $n_{e l}$, and interaction strengths $U / t$.

\begin{tabular}{llllclll}
\hline \hline$M$ & $n_{e l}$ & $U / t$ & $\% E_{\mathrm{UGST}}$ & $\% E_{\mathrm{SUGST}}$ & $\% E_{R J}$ & $\% E_{U J}$ & $\% E_{\mathrm{ref}}$ \\
\hline 16 & 14 & 2 & 97.34 & 97.03 & 99.69 & 97.30 & 96.79 \\
16 & 14 & 4 & 92.81 & 91.70 & 99.02 & 93.07 & 90.75 \\
16 & 14 & 8 & 72.68 & 70.16 & 92.28 & 73.84 & 66.60 \\
16 & 16 & 2 & 80.85 & 80.75 & 99.82 & 93.77 & 93.16 \\
16 & 16 & 4 & 81.84 & 80.18 & 98.57 & 82.61 & 80.24 \\
16 & 16 & 8 & 21.37 & 20.19 & 47.54 & 21.81 & 20.08 \\
36 & 24 & 4 & & & 99.67 & & 98.26 \\
36 & 24 & 8 & & & 98.72 & & 93.53 \\
64 & 28 & 4 & & & 99.74 & & 99.19 \\
64 & 44 & 4 & & & 99.77 & & 98.38 \\
100 & 80 & 2 & 100.00 & 99.98 & & & 99.84 \\
100 & 80 & 4 & 99.85 & 99.61 & & & 97.43 \\
100 & 100 & 2 & 97.69 & 97.56 & & & 97.27 \\
100 & 100 & 4 & 88.50 & 88.08 & & & 87.39 \\
100 & 100 & 6 & 65.70 & 65.19 & & & 64.04 \\
100 & 100 & 8 & 25.01 & 24.76 & & & 23.89 \\
\hline \hline
\end{tabular}




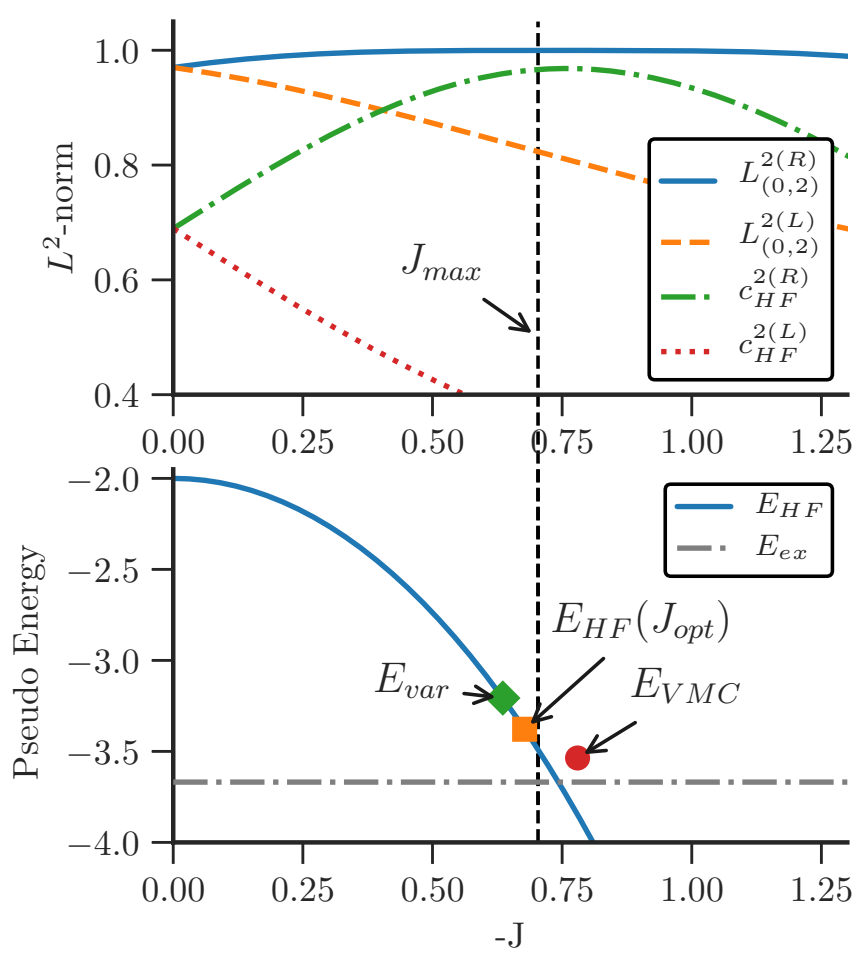

FIG. 1. Top: $L^{2}$ norm of the HF state $c_{H F}^{2(L / R)}$ and within the HF determinant and double excitations, $L_{(0,2)}^{(L / R)}$, for a half-filled 6-site Hubbard chain with periodic boundary conditions at $U / t=4$ and $\mathbf{k}=0$ for the left $\left|\Phi_{0}^{L}\right\rangle$ and right $\left|\Phi_{0}^{R}\right\rangle$ ground-state eigenvector of $\bar{H}$ as a function of $-J$. Bottom: The Hartree-Fock energy $E_{H F}(J)=$ $\left\langle\Phi_{H F}|\bar{H}(J)| \Phi_{H F}\right\rangle$ as a function of $-J$. The dash-dotted line indicates the exact ground-state energy $E_{e x}$ for this system, and since $\bar{H}$ is not Hermitian $E_{H F}$ can drop below the exact energy. Also indicated are the results of minimizing the variance of the energy of the similaritytransformed Hamiltonian $E_{\mathrm{var}}$ by Tsuneyuki [72], the result of solving Eq. (22) $E_{H F}\left(J_{\text {opt }}\right)$, and the result from a VMC optimization $E_{\mathrm{VMC}}$ [96]. The vertical dashed line indicates $J_{\max }$, where $L_{(0,2)}^{2}$ of $\left|\Phi_{0}^{R}\right\rangle$ is maximal. All energies are in units of $t$ and the two panels share the $x$ axis.

$98 \%$ with $E_{R J}$. Off half filling, as can be seen in the $N=$ $36, n_{e l}=24$, and $U / t=8$ case, the relative error remains small even for large interaction. The comparison with the available AFQMC reference results [35,36,73,95], $E_{\text {ref }}$, shows that the solution of Eq. (22) with an on-site Gutzwiller correlator and a restricted Hartree-Fock reference, retrieves above $80 \%$ of the energy for $U / t \leqslant 4$. This gives us confidence that the optimal $J$ obtained by this method is appropriate in the context of the Gutzwiller similarity-transformed Hamiltonian, which we further solve with the FCIQMC method.

\section{Exact diagonalization study}

Due to the non-Hermitian nature of $\bar{H}$ (19) the left and right ground-state eigenvectors $\left|\Phi_{0}^{L / R}\right\rangle$ differ, and depending on the strength of the correlation parameter $J$ they can have a very different form. The most important characteristic for the projective FCIQMC method is the compactness of the sampled wave function. As a measure of this compactness we chose the $L^{2}$ norm of the exact $\left|\Phi_{0}^{L / R}\right\rangle$ contained in the leading HF determinant $\left|\Phi_{H F}\right\rangle$ and double excitations $\left|\Phi_{i j}^{a b}\right\rangle=c_{a}^{\dagger} c_{b}^{\dagger} c_{i} c_{j}\left|\Phi_{H F}\right\rangle$ (spin-index omitted) thereof, i.e., the sum over the squares of the coefficients of these determinants: $L_{(0,2)}^{2}=c_{0}^{2}+\sum_{i<j, a<b} c_{i j a b}^{2}$.

As a simple example, in the top panel of Fig. 1 we show the coefficient of the Hartree-Fock determinant $c_{H F}^{2}$ and $L_{(0,2)}^{2}$ of $\left|\Psi_{0}\right\rangle$ for the 1D half-filled 6-site Hubbard model with periodic boundary conditions at $U / t=4$ and $\mathbf{k}=0$, as a function of the correlation parameter $J . J=0$ corresponds to the original Hamiltonian (18). In the bottom panel of Fig. 1 the HartreeFock energy $E_{H F}$ and results of minimizing the variance of the energy $E_{\mathrm{var}}$ by Tsuneyuki [72], $E_{H F}\left(J_{\mathrm{opt}}\right)$ with $J_{\mathrm{opt}}$ obtained by solving Eq. (22), and variational Monte Carlo (VMC) results [96] $E_{\mathrm{VMC}}$ are shown. Due to the fact that $\bar{H}$ is not Hermitian any more, and hence not bounded by below, $E_{H F}$ can drop below the exact ground-state energy $E_{e x}$, also displayed in Fig. 1, so following Tsuneyuki [72] we termed the energy axis "pseudoenergy." There is a huge increase in $c_{H F}^{2}$ and the $L_{(0,2)}^{2}$ norm of the $\left|\Phi_{0}^{R}\right\rangle$ until an optimal value of $J_{\max }$, close to the $J_{\text {opt }}$ obtained by solving Eq. (22), see Table VI, where $L_{(0,2)}^{2} \approx$ 1 , followed by a subsequent decrease. The result obtained by minimizing the energy variance $E_{\mathrm{var}}$ is higher in energy and farther from $J_{\max }$ than $J_{\mathrm{opt}}$. And, although $E_{\mathrm{VMC}}$ is closer to $E_{e x}$, the optimized correlation parameter obtained by VMC is also farther from $J_{\max }$ than $J_{\mathrm{opt}}$. At the same time $c_{H F}^{2}$ and $L_{(0,2)}^{2}$ of $\left|\Phi_{0}^{L}\right\rangle$ show a monotonic decrease with increasing $-J$. This shows that the amount of relevant information contained within the HF determinant and double excitations thereof can be drastically increased in the right eigenvector, while decreased in the left one. For the calculation of the energy, where only the right eigenvector is necessary, a more efficient sampling with the stochastic FCIQMC method should be possible.

\section{THE FCIQMC METHOD}

The FCIQMC $[75,86]$ method is a projector Monte Carlo method, based on the integrated imaginary-time Schrödinger equation

$$
\frac{\partial|\Psi\rangle}{\partial t}=-\hat{H}|\Psi\rangle \stackrel{\int d t}{\longrightarrow}\left|\Psi_{0}\right\rangle \propto \lim _{t \rightarrow \infty} e^{-t \hat{H}}|\Psi(t=0)\rangle,
$$

where $t$ is an imaginary-time parameter and $|\Psi(t=0)\rangle$ is an arbitrary initial wave function with nonzero overlap with $\left|\Psi_{0}\right\rangle$. One obtains the ground-state energy and wave function by repeatedly applying a first-order difference approximated projector of (25) to the initial state

$$
\left|\Psi_{0}\right\rangle=\lim _{n \rightarrow \infty}\left[\hat{\mathbf{1}}-\Delta t\left(\hat{H}-E_{S} \hat{\mathbf{1}}\right)\right]^{n}|\Psi(0)\rangle,
$$

for $\Delta t<E_{W}^{-1}$ [97], with $E_{W}=E_{\max }-E_{0}$ being the spectral width of $\hat{H}$. If the energy shift $E_{S}=E_{0}$, convergence to a nondiverging and nonzero solution can occur. In practice the shift is dynamically adapted to keep the walker number, explained below, constant, which corresponds to keeping the $L^{1}$ norm of the sampled wave function constant. If the sampled wave function is a stationary solution to the projector, adapting $E_{S}(t)$ to keep the $L^{1}$ norm constant guarantees $E_{S}(t) \rightarrow E_{0}$.

$|\Psi(t)\rangle$ is expanded in an orthonormal basis of $N_{d}$ Slater determinants, $|\Psi(t)\rangle=\sum_{i}^{N_{d}} c_{i}(t)\left|D_{i}\right\rangle$, and the working equation 
for the coefficients $c_{i}$ is

$$
c_{i}(t+\Delta t)=\left\{1-\Delta t\left[H_{i i}-E_{S}(t)\right]\right\} c_{i}(t)-\Delta t \sum_{j \neq i}^{N_{d}} H_{i j} c_{j}(t) .
$$

Equation (27) governs the dynamics of a population of $N_{w}$ signed walkers, which stochastically sample the ground-state wave function $\left|\Psi_{0}\right\rangle$. Since the number of states, $N_{d}$, grows combinatorially with system size, only a stochastic "snapshot" of $\left|\Psi_{0}\right\rangle$ is stored every iteration, where only states occupied by at least one walker are retained. The diagonal term of Eq. (27), $1-\Delta t\left(H_{i i}-E_{S}\right)$, increases or decreases the number of walkers on state $i$. The shift energy $E_{S}(t)$ is dynamically adapted after the chosen number of walkers $N_{w}$ is reached to keep it constant over time. The off-diagonal term, $-\Delta t H_{i j}$, creates new walkers from an occupied determinant $i$ to a connected state $j$. The sum is sampled stochastically by only performing one of these "spawning" events with a probability

$$
p_{\text {spawn }}=\Delta t\left|H_{i j}\right| / p(j \mid i),
$$

and the sign of the new walker is $-\operatorname{sgn}\left(H_{i j}\right)$. At the end of each iteration, walkers with opposite sign on the same determinant, which is a reflection of the fermionic sign problem, are removed from the simulation. For sufficiently many walkers the sign problem can be controlled for many systems. In the intermediate to high interaction regime of the Hubbard model, the number of necessary walkers is proportional to the Hilbert space size, making this "original" FCIQMC method impractical. The initiator approximation i-FCIQMC [75] overcomes this exponential bottleneck at the cost of introducing an initiator bias. It does so by allowing only walkers on determinants above a certain population threshold $n_{\text {init }}$ to spawn onto empty determinants (thereby dynamically truncating the Hamiltonian matrix elements between lowpopulation determinants and empty ones). This is the source of the initiator error, which can be systematically reduced by increasing the walker population. Nevertheless, convergence can be slow, especially if the ground-state wave function is highly spread out over the Hilbert space, as is often the case for strongly correlated systems. On the other hand, convergence can be rapidly obtained if the ground-state eigenvector is relatively compact, and does not require any prior knowledge of this fact, nor of the nature of the compactness. In fact, it is precisely for this reason that the similarity transformations can be of use in the i-FCIQMC method.

In addition to the shift energy $E_{S}(t)$, a projected energy

$$
E_{P}(t)=\frac{\left\langle D_{\mathrm{ref}}|\hat{H}| \Psi(t)\right\rangle}{\left\langle D_{\mathrm{ref}} \mid \Psi(t)\right\rangle}
$$

with $\left|D_{\text {ref }}\right\rangle$ being the most occupied determinant in a simulation, is an estimate of the ground-state energy, if $|\Psi(t)\rangle \approx$ $\left|\Psi_{0}\right\rangle$. An improved estimate (with a smaller variance) can also be obtained by projection onto a multideterminant trial wave function $\left\langle\Phi_{\text {trial }}\right|$,

$$
E_{\text {trial }}(t)=\frac{\left\langle\Phi_{\text {trial }}|\hat{H}| \Psi(t)\right\rangle}{\left\langle\Phi_{\text {trial }} \mid \Psi(t)\right\rangle},
$$

where $\left\langle\Phi_{\text {trial }}\right|$ is obtained as the eigenvector of a small subspace diagonalized similarity-transformed Hamiltonian. This is particularly useful in open-shell problems, where there are several dominant determinants in the ground-state wave function, and as a result $E_{\text {trial }}(t)$ can exhibit notably smaller fluctuations than $E_{P}(t)$.

\section{A. The ST-FCIQMC approach}

In variational approaches the lack of a lower bound of the energy due to the non-Hermiticity of the similaritytransformed Hamiltonian poses a severe problem. As a projective technique, however, the FCIQMC method has no inherent problem sampling the right ground-state eigenvector, obtaining the corresponding eigenvalue by repetitive application of the projector (26). Additionally, the increased compactness of $\left|\Phi_{0}^{R}\right\rangle$ observed in Sec. II C, due to the suppressed double occupations via the Gutzwiller ansatz, tremendously benefits the sampling dynamics of i-FCIQMC. On the other hand, the implementation of the additional 3-body term in (19) necessitate major technical changes to the FCIQMC algorithm. We changed the NECI [98] code to enable triple excitations. Due to momentum conservation and the specific spin relations $(\sigma \sigma \bar{\sigma})$ of the involved orbitals and efficiently analytically calculable 3-body integrals of (19), these could be implemented without a major decrease of the performance of the algorithm. In fact the contractions of the 3-body term in (19), namely $\mathbf{k}=0 \underline{\mathrm{V}}$ $\mathbf{k}^{\prime}=0 \underline{\vee} \mathbf{k}=\mathbf{k}^{\prime} \underline{\vee} \mathbf{q}+\mathbf{k}^{\prime}=\mathbf{s}$, lead to an $\mathcal{O}(M)$ additional cost of the 2-body matrix element, which have the largest detrimental effect on the performance. [There is an $\mathcal{O}\left(M^{2}\right)$ scaling for the diagonal matrix elements, coming from the $\mathbf{k}=\mathbf{k}^{\prime}=\mathbf{0}$ contraction, but this has a negligible overall effect, since we store this quantity for each occupied determinant, and is thus is not computed often.] The additional cost for 2body integrals is similar to the calculation of 1-body integrals in conventional ab initio quantum chemistry calculations and unavoidably hampers the performance, but is manageable. Surprisingly, the performance penalty, due to the additional three-body interactions, decreases with increasing strength of the correlation parameter $J$. This is due to the following fact: the performance of the FCIQMC method depends heavily on the "worst-case" $\left|H_{i j}\right| / p(i \mid j)$ ratio, where $p(i \mid j)$ is the probability to spawn a new walker on determinant $\left|D_{i}\right\rangle$ from $\left|D_{j}\right\rangle$ and $\left|H_{i j}\right|$ is the absolute value of the corresponding matrix element $\left\langle D_{i}|\hat{H}| D_{j}\right\rangle$. The time step $\Delta t$ of the FCIQMC simulation is on-the-fly adapted to ensure the "worst-case" product remains close to unity, $\Delta t\left|H_{i j}\right| / p(i \mid j) \approx 1$. Due to the $M^{2}$ increased number of nonzero matrix elements in the similarity-transformed Hamiltonian (19), the time step $\Delta t$ formally scales as $\mathcal{O}\left(M^{-5}\right)$-momentum conservation decreases the scaling by a factor of $M$-instead of $\mathcal{O}\left(M^{-3}\right)$ for the original Hubbard Hamiltonian (18). However, although numerous, the 3-body terms are easier to calculate and sampled less often, due to their relatively small magnitude and the actually important scaling measure is the necessary number of walkers to achieve a desired accuracy, which is tremendously reduced for the similarity-transformed Hamiltonian.

An optimal sampling in FCIQMC would be achieved, if for every pair $(i, j): p(i \mid j) \approx\left|H_{i j}\right|$ and thus $\Delta t \approx \min \left(1, E_{W}^{-1}\right)$. Since $\bar{H}$ is not Hermitian, the off-diagonal matrix elements are 


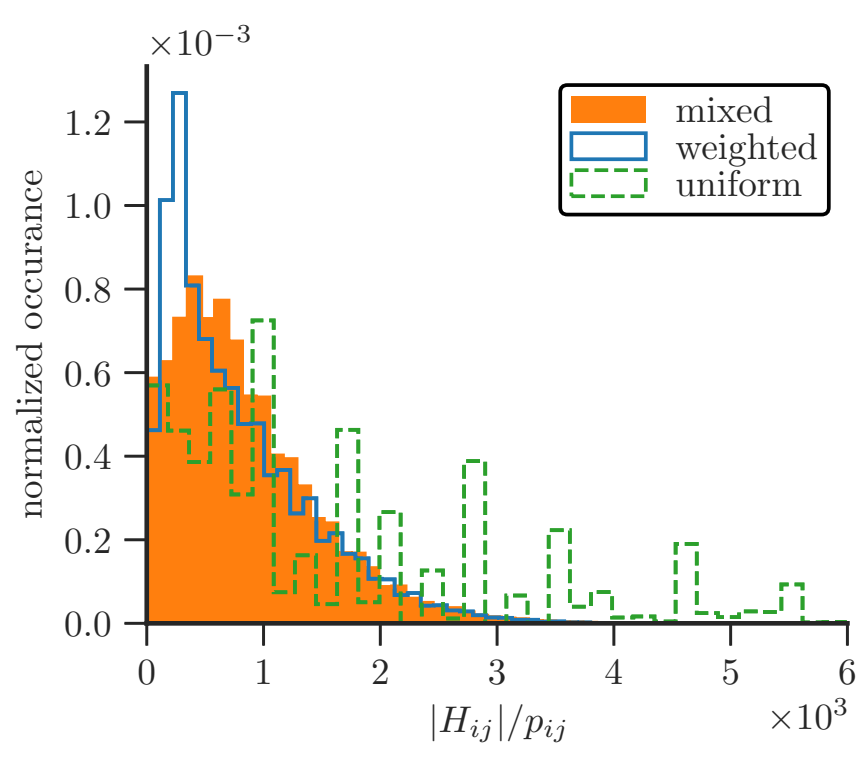

FIG. 2. Histogram of $\left|\bar{H}_{i j}\right| / p_{i j}$ ratios for the half-filled, 50-site, $U / t=4$ Hubbard model with periodic boundary conditions for uniform, weighted, and mixed generation probabilities.

not uniform, as in the original Hamiltonian (18). We therefore need to ensure an efficient sampling by a more sophisticated choice of $p(i \mid j)$. Additionally we can separate $p(i \mid j)$ into a probability to perform a double (triple) excitation $p_{D}\left(1-p_{D}\right)$ since there are still no single excitations in (19), due to momentum conservation. This split into doubles or triples gives us the flexibility, in addition to $\Delta t$, to also adapt $p_{D}$ during run time to bring $\left|H_{i j}\right| / p(i \mid j)$ closer to unity. We observed that with increasing correlation parameter $J$ the dynamically adapted probability to create triple excitations increased, thus reducing the detrimental additional cost to calculate 2-body matrix elements.

When we perform the spawning step in FCIQMC we first decide whether to perform a double excitation with probability $p_{D}$ or a triple excitation with probability $1-p_{D}$. Then we pick two or three electrons $m n(l)$ from the starting determinants $\left(\left|D_{j}\right\rangle\right)$ uniformly, with probability $p_{\text {elec }}$. For a double excitation, due to momentum conservation, we only need to pick one unoccupied orbital, since the second is fixed to fulfill $\mathbf{k}_{m}+\mathbf{k}_{n}=\mathbf{k}_{a}+\mathbf{k}_{b}$. To guarantee $p(i \mid j) \sim\left|H_{i j}\right|$ we loop over the unoccupied orbitals $a$ in $\left|D_{j}\right\rangle$ and create a cumulative probability list with the corresponding matrix elements $\left|H_{i j}(m n, a b)\right|$ and thus pick the specific excitation with $p(i \mid j) \sim\left|H_{i j}\right|$. The cost of this is $\mathcal{O}\left(M^{2}\right)$, due to the loop over the unoccupied orbitals $\sim M$ and the $\mathcal{O}(M)$ cost of the double excitation matrix element calculation. For triple excitations the procedure is similar, except we pick 3 electrons $m_{\sigma}, n_{\sigma}, l_{\bar{\sigma}}$ with $p_{\text {elec }}$; then we pick orbital $a_{\bar{\sigma}}$ of the minority spin uniformly with $p_{a}=1 / n_{\text {holes }}$ and pick orbital $b_{\sigma}$ weighted from a cumulative probability list proportional to $\left|H_{i j}\right|$. The third orbital $c_{\sigma}$ is again determined by momentum conservation $\mathbf{k}_{m}+\mathbf{k}_{n}+\mathbf{k}_{l}=\mathbf{k}_{a}+\mathbf{k}_{b}+\mathbf{k}_{c}$. As opposed to double excitations, this is only of cost $\mathcal{O}(M)$, due to the loop over unoccupied orbitals in $\left|D_{j}\right\rangle$ to determine $b_{\sigma}$. We term this procedure as the weighted excitation generation algorithm.
TABLE III. Ratios of the time step $\Delta t$, time per iteration $t_{\text {iter }}$, and aborted $n_{\text {abort }}$ and accepted excitations $n_{\text {accept }}$ of the different excitation generation probabilities compared to the original $(J=0)$ method with uniform choice of open orbitals as a reference for the half-filled, 50 site, $U / t=4$ Hubbard model with periodic boundary conditions.

\begin{tabular}{llrrrr}
\hline \hline$J$ & Method & $\% \Delta t$ & \multicolumn{1}{c}{$\% t_{\text {iter }}$} & $\% n_{\text {abort }}$ & $\% n_{\text {accept }}$ \\
\hline 0 & weighted & 100.00 & 240.12 & 0.00 & 100.00 \\
$\neq 0$ & uniform & 21.02 & 169.33 & 93.72 & 77.31 \\
$\neq 0$ & mixed & 35.55 & 719.22 & 40.14 & 130.64 \\
$\neq 0$ & weighted & 45.01 & 1506.72 & 0.00 & 165.29 \\
\hline \hline
\end{tabular}

An alternative and simpler algorithm is to pick the unoccupied orbitals in a uniform way. This decreases the cost per iteration, but also leads to a worse worst-case $H_{i j} / p_{i j}$ ratio leading to a decreased time step $\Delta t$. Figure 2 shows the histogram of the $\left|\bar{H}_{i j}\right| / p_{i j}$ ratios for the weighted procedure, described above, the uniform choice of empty orbitals, and a mixed method for the half-filled 50-site Hubbard model at $U / t=4$. In the mixed method, the $\mathcal{O}\left(M^{2}\right)$ scaling double excitations in the weighted scheme are done in a uniform manner, while the $\mathcal{O}(M)$ scaling triple excitations are still weighted according to their matrix element $\left|\bar{H}_{i j}\right|$. Longer tails in a distribution indicate the need for a lower time step to ensure $\Delta t\left|\bar{H}_{i j}\right| / p_{i j} \approx 1$. It is apparent that the mixed scheme possesses the optimal combination of favorable $\left|\bar{H}_{i j}\right| / p_{i j}$ ratios similar to the weighted method, with manageable additional cost per iteration, shown in Table III. Table III shows the relative difference of the time step $\Delta t$, time per iteration $t_{\text {iter }}$, number of aborted excitations $n_{\text {abort }}$, and acceptance rate $n_{\text {accept }}$ of the different methods compared to the original $J=0$ uniform sampled half-filled, 50-site Hubbard model with $U / t=4$. While there is a 7-fold increase of the time per iteration of the mixed scheme compared to the original uniform one, the time step is almost a third larger and the accepted rate of spawning events a third higher. $n_{\text {abort }}$ indicates those spawning attempts which originally are proposed in the uniform scheme, but are finally rejected, due to zero matrix elements, or are Fermi blocked. This quantity is also decreased by more than a half in the mixed method compared to the uniform original scheme. $n_{\text {accept }}$ indicates the number of accepted proposed spawning events and is directly related to $p_{\text {spawn }}(28)$. The choice of the excitation generator is therefore not straightforward and depends on the interaction strength and $J$ : the uniform scheme performs better than expected at small $U / t$, while the mixed scheme performs better at large $U / t$.

\section{RESULTS}

We assessed the performance of initiator ST-FCIQMC (i-ST-FCIQMC) for different Hubbard lattices, as a function of the Gutzwiller correlation factor $J$. As a starting guide for $J$, we use $J_{\text {opt }}$ obtained by solving Eq. (22) for the specific lattice size $M$, number of electrons $n_{e l}$, and interaction strength $U / t$, and calculate the ground-state and excited-state energies with i-ST-FCIQMC. In particular, we were interested in the rate of convergence of the energy with respect to walker number, or in other words, how quickly the initiator error disappeared 


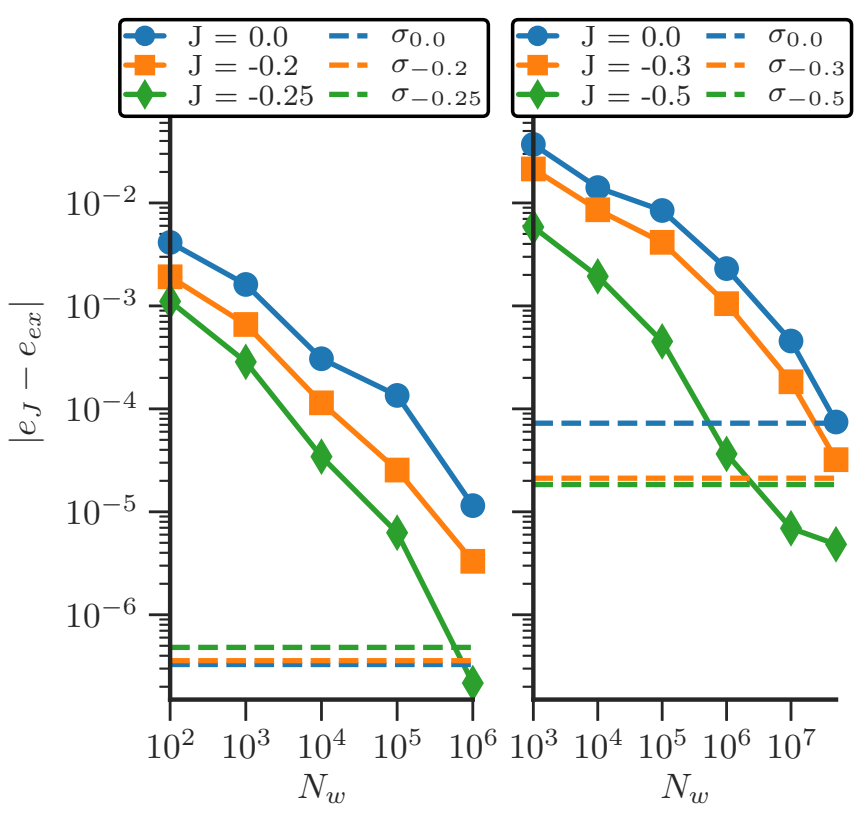

FIG. 3. The error of the energy per site $\left|e_{J}-e_{e x}\right|$ for the halffilled, 18-site Hubbard model for the original $J=0$ and different strengths of the correlation parameter $J$ at $\mathbf{k}=0$ for $U / t=2$ (left) and $U / t=4$ (right) versus the walker number $N_{w}$. The dashed lines indicate the statistical errors of the $N_{w}=10^{6}$ results with $n_{\text {init }}=1.2$ for $U / t=2$ and of the $N_{w}=5 \times 10^{7}$ with $n_{\text {init }}=2.0$ for $U / t=4$. The exact reference results were obtained by Lanczos diagonalization [99].

with increasing walker number. The optimal values of $J$ for each studied system can be found in Table VI in Appendix A. All energies are given per site and in units of the hopping parameter $t$ and the lines in Figs. 3 to 7 are guides to the eye.

\section{A. 18-site Hubbard model}

We first study the 18-site Hubbard model on a square lattice with tilted boundary conditions (see Fig. 8), which can be exactly diagonalized: at half filling and zero total momentum ( $\mathbf{k}=0$ ) it has a Hilbert space of $\sim 10^{8}$ determinants. All the exact reference results were obtained by a Lanczos diagonalization [99]. For this system ST-FCIQMC could be run either in "full" mode or with the initiator approximation, i-STFCIQMC. This enables us to assess to two separate questions, namely the performance of i-ST-FCIQMC with regards to initiator error on the one hand, and compactness of the wave functions resulting from the similarity transformation (without the complicating effects of the initiator approximation), on the other.

Figure 3 shows the error (on a double-logarithmic scale) of the energy per site in the initiator calculation, as a function of walker number. The left panel shows results for the $U / t=2$ system. As one can see there is a steep decrease in the error and even with only $10^{4}$ walkers, for a correlation parameter of $J=-1 / 4$ (close to the $J_{\text {opt }}$ ) the error is below $10^{-4}$. At $10^{6}$ walkers it is well below $10^{-6}$, almost two orders of magnitude lower than the original (i.e., $J=0$ ) Hamiltonian at this value of $N_{w}$. This also confirms the assumption that the chosen ansatz for the correlation function (2) is particularly useful in the low $-U / t$ regime.

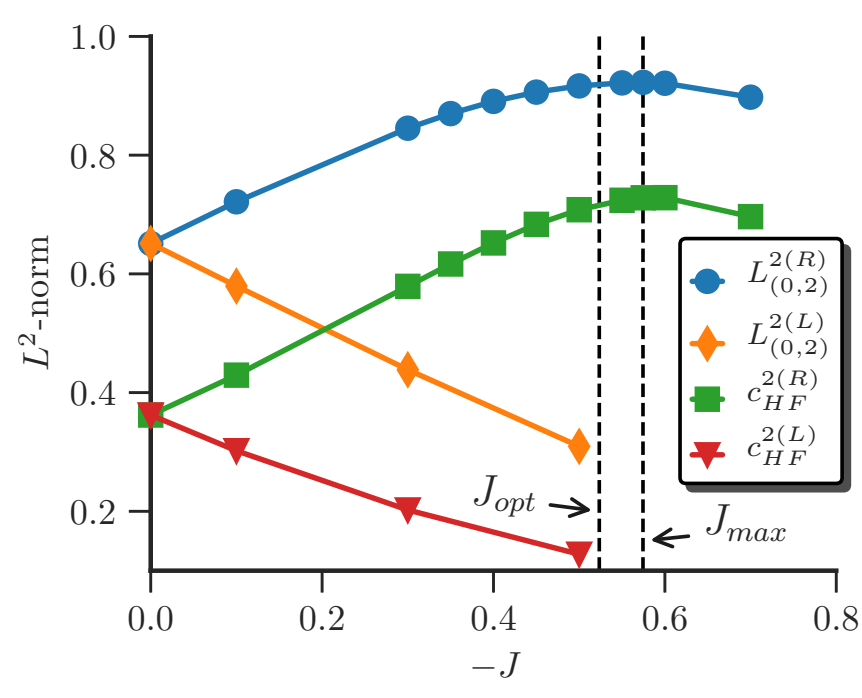

FIG. 4. $L^{2}$ norm captured within the HF determinant $c_{H F}^{2(L / R)}$ and additionally double excitations, $L_{(0,2)}^{2}$, for the half-filled, 18-site Hubbard model at $U / t=4$ for the left and right ground-state eigenvectors of the non-Hermitian similarity-transformed Hamiltonian (19) as a function of $-J$. The results were obtained by a noninitiator STFCIQMC calculation. $\left|\Phi_{L}\right\rangle$ was sampled by running the simulation with positive $J$, which corresponds to conjugating $\bar{H}$. $J_{\max }$ indicates the position of the maximum of $L_{(0,2)}^{2}$ and $J_{\text {opt }}$ is the result of solving Eq. (22).

Results for an intermediate strength, $U / t=4$, are shown in the right panel of Fig. 3. Compared to the $U / t=2$, more walkers are needed to achieve a similar level of accuracy. The two sources for this behavior are as follows.

First, i-FCIQMC calculations on the momentum-space Hubbard model are expected to become more difficult with increasing interaction strength $U / t$, due to the enhanced multiconfigurational character of the ground-state wave function. Second, the chosen correlation ansatz (2) is proven to be more efficient in the low $U / t$ regime [46]. Nevertheless, the results shown in Fig. 3 show a steep decrease in the doublelogarithmic plot of the error with increasing walker number. The decrease is steeper for $J=-1 / 2$, close to the analytic result obtained with $J_{\mathrm{opt}}=-0.5234470$. For $J=-1 / 2$, at walker numbers up to $5 \times 10^{7}$, we are to within error bars at the exact result. At a walker number of $10^{7}$ there is a two orders of magnitude difference in the error of the $J=-1 / 2$ and $J=0$ result.

To confirm the more compact form of the right groundstate eigenvector, mentioned in Sec. II C, we performed two analyses. First was the study of the $L^{2}$ norm captured within the HF determinant $c_{H F}^{2(L / R)}$ and additionally double excitations, $L_{(0,2)}^{2}$, for the ST-FCIQMC wave function. In Fig. 4 $L_{(0,2)}^{2}$ of the left and right ground-state eigenvectors of the half-filled, 18-site, $U / t=4$ Hubbard model as a function of $-J$ are shown. The results were obtained by running full noninitiator ST-FCIQMC calculations to avoid any influence of the initiator error. The left eigenvector was obtained by running with positive $J$, which corresponds to a conjugation of $\bar{H}$,

$$
\bar{H}(J)^{\dagger}=\left(e^{-\hat{\imath}} \hat{H} e^{\hat{\imath}}\right)^{\dagger}=e^{\hat{\imath}} \hat{H} e^{-\hat{\tau}}=\bar{H}(-J),
$$




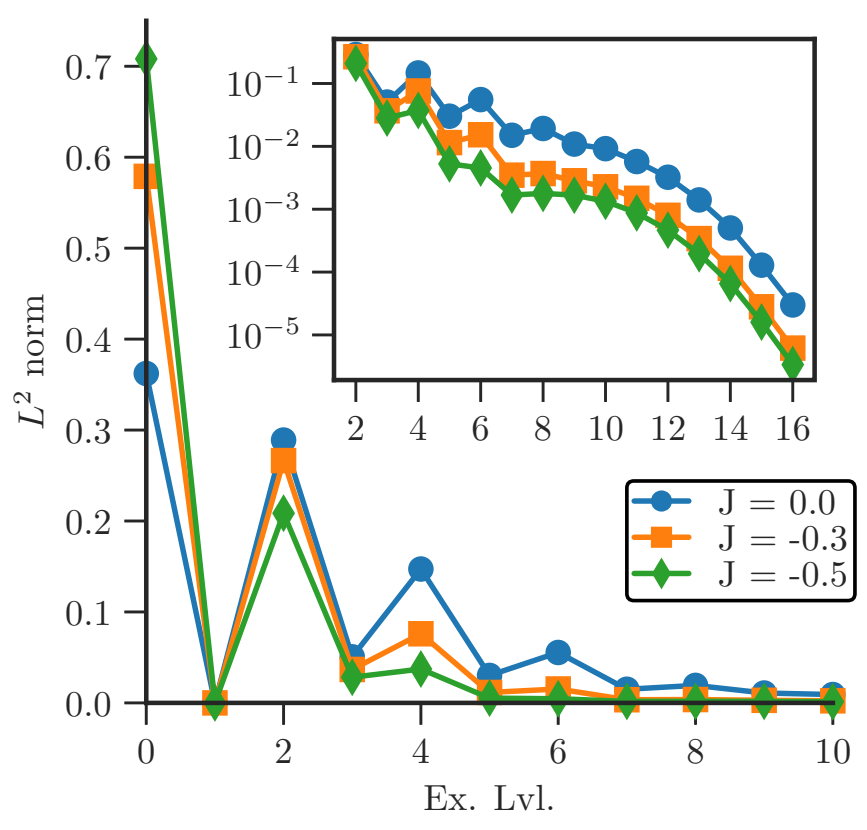

FIG. 5. The $L^{2}$ norm contained in each excitation level relative to the HF determinant, indicated by excitation level 0 , for the half-filled, 18 -site, $U / t=4, \mathbf{k}=0$ Hubbard model for different values of $J$. The inset shows the tail of the same data on a logarithmic scale.

since $\hat{H}^{\dagger}=\hat{H}$ and $\hat{\tau}^{\dagger}=\hat{\tau}$. And,

$$
\bar{H}^{\dagger}\left|\Phi_{L}\right\rangle=E\left|\Phi_{L}\right\rangle, \quad \text { with } \quad\left|\Phi_{L}\right\rangle=e^{-\hat{\tau}}|\Psi\rangle .
$$

Similarly to the exact results for the 6-site model in Fig. 1, the right eigenvector shows a huge compactification compared to the original $J=0$ result, going from 0.65 to over 0.9 for $L_{(0,2)}^{2}$. The "optimal" value of $J=J_{\max }=-0.57444831$, where $L_{(0,2)}^{2}$ is maximal, is close to the analytically obtained $J_{\text {opt }}=-0.5234470$, indicating that we can simply use $J_{\text {opt }}$ without further numerical optimization of $J$, and still be close to optimal conditions.

Figure 5 shows the $L^{2}$ norm contained in each excitation level relative to the HF determinant for the half-filled, 18site, $U / t=4$ Hubbard model for different values of $J$. For $J=-1 / 2$ there is a huge increase in the $L^{2}$ norm of the HF determinant, indicated by an excitation level of 0 , while it drops off very quickly for higher excitation levels and remains one order of magnitude lower than the $J=0$ result above an excitation level of 5 .

Our second analysis on the compactness of $\left|\Phi_{0}^{R}\right\rangle$ consisted of running truncated CI [100] calculations, analogous to the CISD, CISDTQ, etc., methods of quantum chemistry. Here we truncate the Hilbert space by only allowing excitation up to a chosen value $n_{\text {trunc }}$ relative to the HF determinant. Figure 6 shows the error of the energy per site as a function of $n_{\text {trunc }}$ for different $J$. For $J=-1 / 2$ we are below $10^{-4}$ accuracy already at only quintuple excitation, which is two orders of magnitude lower than the original $J=0$ result at this truncation level. The error bars in the inset of Fig. 6 are from the $n_{\text {trunc }}=8$ simulations for each value of $J$, which do not differ much from $n_{\text {trunc }}=5$ to $n_{\text {trunc }}=8$ for each simulation. Already at sextuple excitations we are well within error bars of

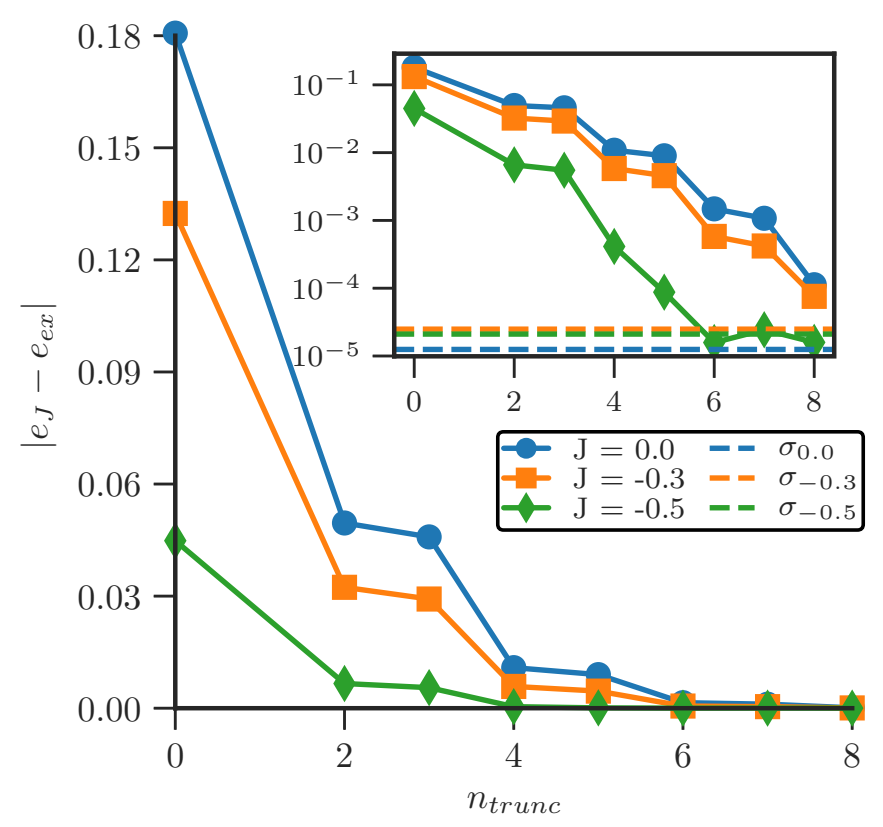

FIG. 6. Error of the energy per site versus the excitation level truncation $n_{\text {trunc }}$ in the half-filled, 18-site, $U / t=4, \mathbf{k}=0$ Hubbard model for different values of $J$. The inset shows the absolute error on a logarithmic scale. The dashed lines in the inset indicate the statistical error for the $n_{\text {trunc }}=8$ results for each value of $J$.

the exact result for $J=-1 / 2$, with an error that is two orders of magnitude smaller than the $J=0$ result.

\section{Off-half-filling $14 e^{-}$in 18 sites}

We have also investigated the applicability of the i-STFCIQMC method to the off-half-filling case, and also to excited-state calculations. To this end we calculated the ground, first, and second excited states of the $14 e^{-}$in the 18 -site, $U / t=4, \mathbf{k}=0$ system. Such a system can be prepared by removing 4 electrons ( $2 \alpha$ and $2 \beta$ spins) from the corners of the Fermi-sea determinant, and using this as a starting point for an i-ST-FCIQMC simulation. Excited states are obtained by running multiple independent runs in parallel and applying a Gram-Schmidt orthogonalization to a chosen number of excited states [101]

$$
\left|\Phi_{i}(t+\Delta t)\right\rangle=\hat{P}_{i}(t+\Delta t)\left[\mathbf{1}-\Delta t\left(\hat{H}-E_{S, i}\right)\right]\left|\Phi_{i}(t)\right\rangle,
$$

with $\hat{P}_{i}(t)$ being the orthogonal projector

$$
\hat{P}_{i}(t)=\mathbf{1}-\sum_{j<i} \frac{\left|\Phi_{j}(t)\right\rangle\left\langle\Phi_{j}(t)\right|}{\left\langle\Phi_{j}(t) \mid \Phi_{j}(t)\right\rangle} \quad \text { with } \quad E_{j}<E_{i} .
$$

However, since the set of right eigenvectors $\left|\Phi_{i}^{R}\right\rangle$ of a nonHermitian operator are not guaranteed to be orthogonal, we cannot rely on the projected energy estimate (29) as an estimate for the excited state energy. By orthogonalizing each eigenvector $\left\langle\Phi_{i}^{E} \mid \Phi_{j}^{R}\right\rangle \stackrel{!}{=} 0$ for $i \neq j$ ( $i$ and $j$ indicate the excited states), the sampled excited states will in general not be identical to the exact right eigenvectors of $\bar{H}$. On the other hand, since the spectrum of $\bar{H}$ does not change due to the similarity transformation (6), the shift energy $E_{S}$ in (33), 
(a) $E_{0}$

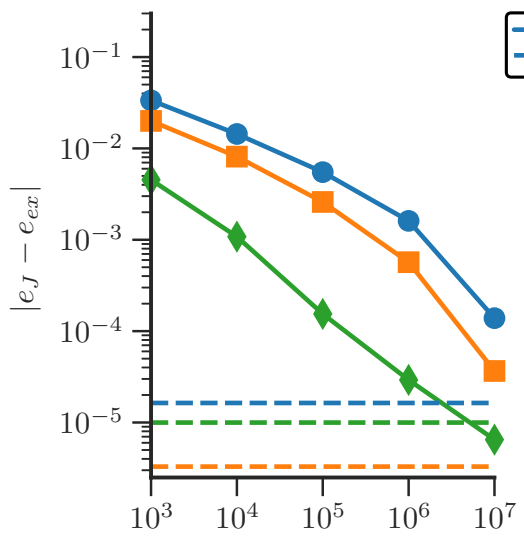

(b) $E_{1}$

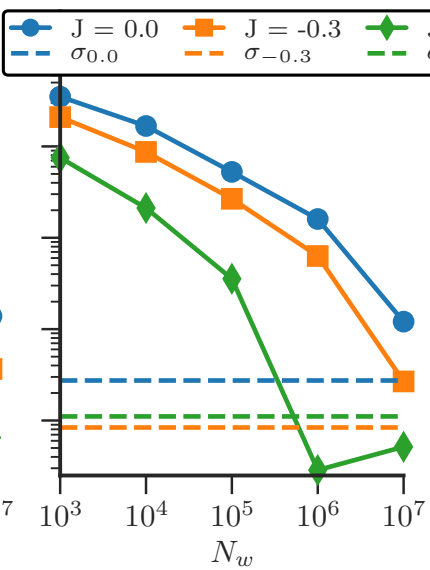

(c) $E_{2}$

FIG. 7. Energy per site error compared to exact Lanczos results [99] for the $14 e^{-}$in the 18 -site, $U / t=4, \mathbf{k}=0$ Hubbard model for the (a) ground, (b) first excited, and (c) second excited state as a function of walker number $N_{w}$. All three panels share the $x$ and $y$ axes. The dashed lines indicate the statistical errors of the $N_{w}=10^{7}$ for each value of $J$.

dynamically adapted to keep the walker number constant, remains a proper estimate for the excited-state energy. This interesting fact is developed further in Appendix C. Additionally, if the excited state belongs to a different spatial or total-spin symmetry sector the overlap with the ground state is zero, so our excited-state approach within the FCIQMC formalism, via orthogonalization, correctly samples these orthogonal excited states.

Figure 7 shows the energy per site error of the ground, first, and second excited states of the $14 e^{-}$in the 18-site, $U / t=4, \mathbf{k}=0$ system, compared to exact Lanczos reference results [99] for different values of $J$ versus the walker number $N_{w}$, obtained via the shift energy $E_{S, i}$. All states show a similar behavior of the energy per site error. The closer $J$ gets to the optimal value of $J_{\mathrm{opt}}=-0.557941$ for $U / t=4$, which is determined for $E_{0}$; one observes that more than an order of magnitude fewer walkers are necessary to achieve the same accuracy as the $J=0$ case. This is true for all the states considered. For $E_{1}$, the energy difference of the $N_{w}=10^{7}$ and $J=-1 / 2$ calculation is already within the statistical error of $10^{-5}$, hence the nonmonotonic behavior. The size of the absolute error of these states is comparable to the absolute error of the half-filled, 18 -site, $U / t=4$ system, shown in the right panel of Fig. 3. Since, without a chemical potential, the total ground-state energy per site of the $n_{e l}=14$ system, $e_{0}^{(14)}=-1.136437$, is lower than the half-filled one, $e_{0}^{(18)}=-0.958466$, the relative error is in fact smaller off half filling. As already mentioned above and shown in Tables I and II, the projective solution based on the restricted HartreeFock determinant (22) also yields smaller relative errors off half filling. These results give us confidence to also apply the i-ST-FCIQMC method to systems off half filling and for excited-state energy calculations.

\section{Symmetry analysis}

As mentioned above, the set of right eigenvectors of a non-Hermitian operator is in general not orthogonal, except when the eigenvectors belong to different irreducible representations and/or total spin symmetry sectors. Here we investigate the interesting influence of the similarity transformation on the symmetry properties of the truncated lowenergy subspace of the $14 e^{-}$in the 18 -site system with total $\mathbf{k}=0$. There are 8 important low-energy determinants with the 5 lowest energy $\mathbf{k}$ points double occupied and $4 e^{-}$ distributed among the 4 degenerate orbitals of the corner of the square $\mathbf{k}_{1}=(-1,-1), \mathbf{k}_{2}=(1,-1), \mathbf{k}_{3}=(-1,1)$, and $\mathbf{k}_{4}=(1,1)$ to preserve the total $\mathbf{k}=0$ symmetry. This is illustrated in Fig. 8, where filled red circles indicate the doubly occupied $\mathbf{k}$ points and half-filled green circles the singly occupied ones. The point group of the square lattice is $D_{4 h}$. There are 2 closed-shell determinants in this set, with opposite

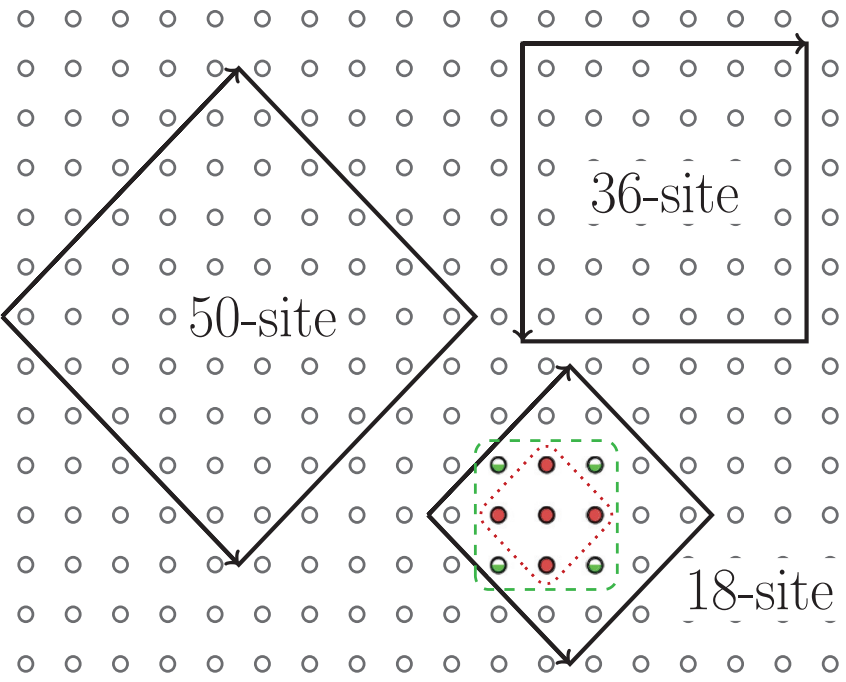

FIG. 8. The three different square lattices studied in this paper. The 18- and 50-site lattices have tilted periodic boundary conditions with lattice vectors $\mathbf{R}_{1}=(3,3), \mathbf{R}_{2}=(3,-3)$ and $\mathbf{R}_{1}=$ $(5,5), \mathbf{R}_{2}=(5,-5)$, respectively. The 36-site lattice is studied with periodic and mixed, periodic along the $x$ axis and antiperiodic boundary conditions along the $y$ axis. The filled red circles in the 18-site lattice indicate the doubly occupied states and the half-filled green circles the singly occupied states in the subspace studied in Sec. IV A 2. 
TABLE IV. Irreducible representations and spin symmetry of the ground state $E_{0}$ and first two excited states $E_{1}, E_{2}$ of the $U / t=4$, $\mathbf{k}=0,14 e^{-}$in the 18-site Hubbard model for the full and subspace solutions for different values of $J$. For a large enough correlation parameter $J$ the ground state of the low-energy subspace resembles the correct symmetry structure as for the full solution.

\begin{tabular}{lccc}
\hline \hline & $E_{0}$ & $E_{1}$ & $E_{2}$ \\
\hline Full & ${ }^{1} A_{1 g}$ & ${ }^{5} B_{1 g}$ & ${ }^{1} B_{2 g}$ \\
$J=0$ subspace & ${ }^{5} B_{1 g}$ & ${ }^{1} A_{1 g}$ & ${ }^{1} B_{2 g}$ \\
$J=-0.72$ subspace & ${ }^{1} A_{1 g}$ & ${ }^{5} B_{1 g}$ & ${ }^{1} B_{2 g}$ \\
\hline \hline
\end{tabular}

k points doubly occupied and 6 open-shell determinants with all 4 corners of the Brillouin zone singly occupied. Without a correlation parameter all these 8 determinants are degenerate in energy, while with $J \neq 0$ this degeneracy is lifted. To study the low-energy properties of this system we diagonalized $\bar{H}$ in this subspace. Table IV shows the results. We found that with $J=0$ the ground state of this subspace has a different spatial and spin symmetry, ${ }^{5} B_{1 g}$, than the ground state of the full system, which belongs to ${ }^{1} A_{1 g}$. At approximately $J \approx-0.71$ there is a crossover and the subspace ground state changes to ${ }^{1} A_{1 g}$ symmetry. The first excited state in the subspace is then the ${ }^{5} B_{1 g}$, which is also the symmetry of the first excited state of the full system and the second excited state is of ${ }^{1} B_{2 g}$ symmetry, the same as the second excited state of the not truncated system. Therefore the similarity transformation not only ensures a more compact form of the ground- and excited-state wave functions, but also correctly orders the states obtained from subspace diagonalizations. The implication is that, in the off-half-filling Hubbard model, the structure of the ground state has very important contributions arising from high-lying determinants, so much so that they are necessary to get a qualitatively correct ground-state wave function (i.e., one with the correct symmetry and spin). With the similarity-transformed Hamiltonian, however, this is not the case. Even small subspace diagonalizations yield a ground-state wave function with the same symmetry and spin as the exact one. In other words, the similarity transformation effectively downfolds information from higher-lying regions of the Hilbert space to modify the matrix elements between the low-lying determinants. Since the structure of the groundstate eigenvector already has the correct symmetry (and therefore signs) in small subspaces, the rate of convergence of the solution with respect to the addition of further determinants is much more rapid. We believe this is a crucial property which leads to the observed greatly improved convergence rate of the i-ST-FCIQMC method in the off-half-filling regime.

\section{B. Results for the 36- and 50-site Hubbard models}

To put the i-ST-FCIQMC method to a stern test, we applied it to two much larger systems, namely 36-site and 50-site lattices, which are well beyond the capabilities of exact diagonalization. In the case of the 36 -site $(6 \times 6)$ lattice, we considered two boundary conditions, namely fully periodic (PBC) and a mixed periodic-antiperiodic (along the $x$ and $y$ axes, respectively), the latter being used in some studies to avoid degeneracy of the noninteracting solution [11]. We considered two fillings, namely half filling and $24 e^{-}$, at $U / t=$ 2 and $U / t=4$. The optimal $J_{\text {opt }}$ was determined by solving Eq. (22) and is listed in Table VI in Appendix A. For the $6 \times 6$ by lattice we compared our results to AFQMC calculations [36], which are numerically exact at half filling [93]. The results are shown in Table V. While the original i-FCIQMC method shows a large error even at walker numbers up to $N_{w}=5 \times 10^{8}$ the i-ST-FCIQMC method agrees with the AFQMC reference to within $1 \sigma$ error bars in all but one case ( $\operatorname{PBC} U / t=4$ half filled), where the agreement is within $2 \sigma$. Even in that case the energies agree to better than $99.8 \%$. The

TABLE V. Zero-temperature, $\mathbf{k}=0$ ground-state energy results for the 36-site and 50-site Hubbard models for various interaction strengths $U / t$, numbers of electrons $n_{e l}$, and periodic (PBC) and mixed (anti)periodic boundary conditions along the (y) $x$ axis, obtained with the initiator FCIQMC and the i-ST-FCIQMC method compared with available (CP-)AFQMC and linearized-AFQMC reference results [36,95,106-108]. The differences from the AFQMC reference energies are displayed as $\Delta E$. The correlation parameter $J$ was chosen close to the optimal $J_{\text {opt }}$ obtained by solving Eq. (22) listed in Table VI of Appendix A for the specific $U / t$ value. An initiator threshold of $n_{\text {init }}=2.0$ was chosen and convergence of the energy up to a walker number of $N_{w}=10^{9}$ was checked.

\begin{tabular}{|c|c|c|c|c|c|c|c|c|}
\hline$M$ & $U / t$ & $n_{e l}$ & $\mathrm{BC}$ & $E_{\text {ref }}$ & i-FCIQMC & $\Delta E_{J=0}$ & i-ST-FCIQMC & $\Delta E_{J}$ \\
\hline 36 & 4 & 24 & PBC & $-1.18525(4)$ & $-1.182003(57)$ & $0.003247(97)$ & $-1.1852109(52)$ & $0.000039(45)$ \\
\hline 36 & 2 & 36 & PBC & $-1.15158(14)$ & $-1.149734(95)$ & $0.00185(24)$ & $-1.151544(18)$ & $0.00004(16)$ \\
\hline 36 & 4 & 36 & APBC & $-0.87306(56)$ & $-0.847580(84)$ & $0.025480(64)$ & $-0.872612(50)$ & $0.00045(61)$ \\
\hline 36 & 4 & 36 & PBC & $-0.85736(25)$ & $-0.82807(87)$ & $0.0293(11)$ & $-0.85625(30)$ & $0.00111(55)$ \\
\hline 50 & 2 & 50 & PBC & $-1.22278(17)$ & $-1.220590(16)$ & $0.00219(19)$ & $-1.2228426(80)$ & $-0.00006(18)$ \\
\hline 50 & 3 & 50 & PBC & $-1.03460(30)$ & $-1.023064(35)$ & $0.01154(34)$ & $-1.034788(18)$ & $-0.00019(32)$ \\
\hline 50 & 4 & 50 & PBC & $-0.879660(20)$ & $-0.83401(15)$ & $0.04565(17)$ & $-0.880657(60)$ & $-0.000997(80)$ \\
\hline 50 & 4 & 48 & $\mathrm{PBC}$ & $-0.93720(15)$ & $-0.89610(12)$ & $0.04110(27)$ & $-0.93642(40)$ & $0.00078(55)$ \\
\hline 50 & 4 & 46 & $\mathrm{PBC}$ & $-0.9911420(86)$ & $-0.95550(15)$ & $0.03564(24)$ & $-0.990564(89)$ & $0.00058(18)$ \\
\hline 50 & 4 & 44 & PBC & $-1.037883(59)$ & $-1.006483(38)$ & $0.031400(97)$ & $-1.037458(47)$ & $0.00043(11)$ \\
\hline
\end{tabular}


TABLE VI. $J_{\text {opt }}$ obtained by solving Eq. (22) for the specific lattice sizes, fillings, and $U / t$ values used in this paper. $J_{e x}$ is the value that sets the $J$-dependent Hartree-Fock energy, $E_{J}^{H F}$, to the exact energy, if available, or to the AFQMC reference energies for larger systems.

\begin{tabular}{lcccccc}
\hline \hline$M$ & $U / t$ & $n_{e l}$ & $J_{\mathrm{opt}}$ & $J_{e x}$ & $e_{e x}$ & $e_{J}$ \\
\hline 6 & 4 & 6 & -0.67769 & -0.74282 & -0.61145 & -0.56306 \\
18 & 2 & 18 & -0.27053 & -0.28536 & -1.32141 & -1.31697 \\
18 & 4 & 18 & -0.52345 & -0.57472 & -0.95847 & -0.92697 \\
18 & 4 & 14 & -0.55794 & -0.62474 & -1.13644 & -1.09786 \\
36 & 2 & 36 & -0.30485 & -0.45423 & -1.15158 & -1.09840 \\
$36^{\mathrm{a}}$ & 2 & 36 & -0.28683 & -0.31783 & -1.20831 & -1.19904 \\
36 & 4 & 36 & -0.58521 & -0.79141 & -0.85736 & -0.71675 \\
$36^{\mathrm{a}}$ & 4 & 36 & -0.55295 & -0.65181 & -0.87306 & -0.81145 \\
$36^{\mathrm{a}}$ & 4 & 24 & -0.53570 & & & -1.13399 \\
$36^{\mathrm{b}}$ & 4 & 24 & -0.52372 & -0.57014 & -1.18530 & -1.16457 \\
50 & 1 & 50 & -0.14290 & -0.15357 & -1.43718 & -1.43561 \\
50 & 2 & 50 & -0.28298 & -0.30852 & -1.22278 & -1.21523 \\
50 & 3 & 50 & -0.41788 & -0.46639 & -1.03460 & -1.01278 \\
50 & 4 & 50 & -0.54600 & -0.63177 & -0.87966 & -0.82601 \\
50 & 4 & 48 & -0.54945 & -0.62810 & -0.93720 & -0.88954 \\
50 & 4 & 46 & -0.55208 & -0.62227 & -0.99114 & -0.95008 \\
50 & 4 & 44 & -0.54772 & -0.61530 & -1.03788 & -1.00016 \\
50 & 4 & 42 & -0.54324 & -0.60263 & -1.08002 & -1.047 \\
50 & 4 & 26 & -0.51076 & -0.56162 & -1.11564 & -1.09946 \\
\hline \hline
\end{tabular}

a Antiperiodic $\mathrm{BC}$ along $y$ axis.

${ }^{\mathrm{b}}$ Open-shell $\mathbf{k}=0$ reference.

small discrepancy could be due to this system being strongly open shell, making equilibration more challenging.

The 50-site Hubbard lattice corresponds to a $5 \sqrt{5} \times 5 \sqrt{5}$ tilted square, which has been widely investigated using the AFQMC method. We considered half-filling and various off-half-filling, $n_{e l}=26,42,44,46$, and 48 cases for $U / t=$ $1,2,3$, and 4 and calculated the ground-state energy. The optimal $J$ are listed in Table VI in Appendix A. This system size, especially with increasing $U / t$ and off half filling, was previously unreachable with the FCIQMC method. We compare our half-filling results to AFQMC $[93,102,103]$ reference values, which do not have a sign problem at half filling [93]. The remaining sources of error are extrapolation to zero temperature and finite steps, both of which are expected to be very small. Off half filling, exact AFQMC results are not available, and we compare against constrained-path AFQMC (CP-AFQMC) [104,105] and linearized AFQMC (L-AFQMC) [106].

Table $\mathrm{V}$ shows the results for various fillings and $U / t$ values of the reference calculations, the original i-FCIQMC and the i-ST-FCIQMC methods. We converged our results for this system size up to a walker number of $N_{w}=10^{9}$. We can see that the original i-FCIQMC method performs well for the weakly correlated half-filled $U / t=1$ system, but fails to reproduce the reference results at $U / t=2$ for this system size, and the discrepancy worsens with increasing interaction. The i-ST-FCIQMC method on the other hand agrees within error bars with the reported reference calculation up to $U / t=3$ at half filling. Similarly to the half-filled 36-site lattice, the iST-FCIQMC results are slightly below the AFQMC reference results at $U / t=4$, which could be a finite-temperature effect of the AFQMC reference results.

We investigated the half-filled, 50-site, $U / t=4$ system further by performing the convergence of a truncated CI expansions, similarly to the 18 -site lattice. The results are shown in Fig. 9. The convergence with excitation level truncation shows that convergence occurs from above, and at 6-fold excitations we are converged to statistical accuracy to the fully unconstrained simulation. The energy at 6-fold truncation is indeed slightly below the AFQMC result, although the discrepancy is small (approximately $0.1 \%$ ). It is intriguing that the CI expansion of the 50-site lattice is converged at 6fold excitations, which is the same as observed for the 18-site lattice. This suggests that linear solutions to the similaritytransformed Hamiltonian may be size-consistent to a greater

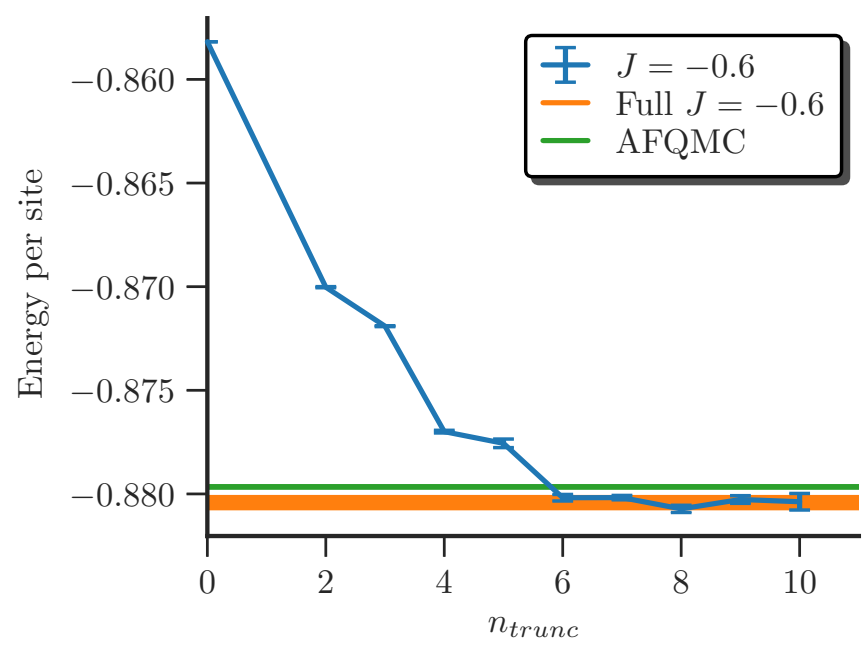

FIG. 9. The energy per site versus the excitation level truncation $n_{\text {trunc }}$ in the half-filled, 50-site, $U / t=4, \mathbf{k}=0$ Hubbard model. AFQMC reference [108] and nontruncated i-ST-FCIQMC results are also shown. Similarly to the 18-site system at half-filling (Fig. 6), the energies are well converged at 6-fold excitations. 
degree than similar truncations to the original untransformed Hamiltonian. This question however is left for a future study.

For $U / t=4$ off half filling the i-ST-FCIQMC energies are consistently slightly above the reference AFQMC results. However the approximations in the off-half-filling AFQMC calculations can lead to energies below the exact ones. For example [95], CP-AFQMC on a $4 \times 4$ lattice with $14 e^{-}$ and $U / t=4$ gives an energy of -0.9863 (1) compared to an exact energy of -0.9840 , i.e., an overshoot of $0.2 \%$. Similar overshoots are observed at other fillings. In the off-halffilling regime in the 50-site system at $U / t=4$, CP-AFQMC overshoots our i-ST-FCIQMC results by similar amounts. Therefore our results are in line with ED results for smaller lattices, and thus represent a new set of benchmarks for the off-half-filling 50-site Hubbard model.

\section{DISCUSSION, CONCLUSION, AND OUTLOOK}

We have used a projective solution based on the restricted Hartree-Fock determinant to obtain an optimized Gutzwiller correlation parameter. For low to intermediate interaction strength, this method generally recovers over $80 \%$ of the ground-state energy. Based on this mean-field solution we derived a similarity-transformed Hubbard Hamiltonian, generated by the Gutzwiller ansatz, in a momentum-space basis. We solved for the exact ground- and excited-state energies of this non-Hermitian operator with the FCIQMC method. We have shown that the right eigenvector of the non-Hermitian Hamiltonian has a dramatically more compact form, due to suppression of energetically unfavorable double occupancies, via the Gutzwiller ansatz. This increased compactness of the right eigenvectors allowed us to solve the Hubbard model for system sizes, which were previously unreachable with the i-FCIQMC method. We benchmarked our results with highly accurate AFQMC reference results and find extremely good agreement at and off half filling up to interaction strengths of $U / t=4$. We hope this combination of a similarity transformation based on a correlated ansatz for the ground-state wave function and subsequent beyond mean-field solution with FCIQMC can aid the ongoing search for the phase diagram of the two-dimensional Hubbard model in the thermodynamic limit.

An important extension of the present work will be to compute observables other than the energy. To compute the expectation values of operators $\hat{O}$ which do not commute with the Hamiltonian we need additionally to obtain the left eigenvector of the non-Hermitian $\bar{H}$ with the ansatz $\left\langle\Psi_{L}\right|=$ $\langle\Phi| e^{-\hat{\tau}}$ :

$$
\langle\Psi| \hat{H}=\langle\Phi| e^{-\hat{\tau}} H=E\langle\Phi| e^{-\hat{\tau}} .
$$

The expectation value of the similarity-transformed operator $\bar{O}=e^{-\hat{\imath}} \hat{O} e^{\hat{\imath}}$ with $\left|\Phi_{R / L}\right\rangle$ yields the desired

$$
\frac{\left\langle\Phi_{L}|\bar{O}| \Phi_{R}\right\rangle}{\left\langle\Phi_{L} \mid \Phi_{R}\right\rangle}=\frac{\left\langle\Psi\left|e^{\hat{\imath}} e^{-\hat{\imath}} \hat{O} e^{\hat{\imath}} e^{-\hat{\imath}}\right| \Psi\right\rangle}{\left\langle\Psi\left|e^{\hat{\imath}} e^{-\hat{\imath}}\right| \Psi\right\rangle}=\langle\hat{O}\rangle .
$$

As already observed in Sec. IV A, applying $\bar{H}$ with $-J$ yields the left eigenvector $\left|\Phi_{L}\right\rangle=e^{\hat{\tau}}|\Psi\rangle$. To perform this in the FCIQMC we only need to run two independent simulations in parallel, as is already done in replica-sampling of reduced density matrices [109], where the two runs use an opposite sign of the correlation parameter $J$. Observables, $\hat{O}$, which commute with the chosen Gutzwiller correlator $[\hat{\tau}, \hat{O}]=0$, such as the double occupancy $\left\langle n_{\uparrow} n_{\downarrow}\right\rangle$, can be calculated by the 2-body reduced density matrix obtained with the left and right eigenvector

$$
\Gamma_{p q, r s}=\left\langle\Phi_{L}\left|c_{p}^{\dagger} c_{q}^{\dagger} c_{s} c_{r}\right| \Phi_{R}\right\rangle,
$$

with normalized $\left\langle\Phi_{L} \mid \Phi_{R}\right\rangle=1$ and $p, q, r$, and $s$ denoting spin-orbital labels in the momentum space. Noncommuting observables, $[\hat{\tau}, \hat{O}]$, have to be similarity-transformed $\bar{O}=$ $e^{-\hat{\imath}} \hat{O} e^{\hat{\imath}}$ and might require higher-order density matrices.

Simultaneous calculation of the left eigenvectors $\left|\Phi_{L}^{i}\right\rangle$ also allows us to obtain the correct excited-state wave functions, in addition to the already-correct excited-state energy via the shift energy $E_{S, i}$ mentioned in Sec. IV A 1 and Appendix C, in the following manner: For $m$ excited states we run $2 m$ simulations in parallel, where every odd numbered calculation solves for a right eigenstate $\left|\Phi_{R}^{i}\right\rangle$, which is orthogonalized against all $\left|\Phi_{L}^{j}\right\rangle$ with $E_{j}<E_{i}$. And vice versa, every even numbered simulation solves for a left eigenvector $\left|\Phi_{L}^{i}\right\rangle$, orthogonalized against each $\left|\Phi_{R}^{j}\right\rangle$ with $E_{j}<E_{i}$. In this shoelace manner $m$ left and right excited state eigenvectors are obtained based on the biorthogonal property of left and right eigenvectors of non-Hermitian operators $\left\langle\Phi_{L}^{i} \mid \Phi_{R}^{j}\right\rangle=0$ for $i \neq j$. Results on observables other than the energy and correct left and right eigenvectors of excited states will be reported in future work.

To perform accurate thermodynamic-limit extrapolations, we also need to reduce the finite-size errors of the kinetic term in Eq. (7). This can be done by twist-averaged boundary conditions [36,110-112], which are readily applicable for the similarity-transformed Hamiltonian in FCIQMC, and will be reported in future work.

\section{ACKNOWLEDGMENTS}

The authors thank Dr. Olle Gunnarsson for providing the 18-site exact diagonalization results, Dr. Pablo R. Lopez for the VMC correlation parameter optimization, and Kai Guther for helpful discussions.

\section{APPENDIX A: OPTIMIZATION OF $J$}

As mention in Sec. II B, similarly to the optimization of coupled-cluster amplitudes [113], we want to solve for the single parameter $J$ of the ansatz (2) by projection. Projecting the ansatz on $\left\langle\Phi_{H F}\right|$ would yield us the energy $E_{J}^{H F}$ :

$$
\left\langle\Phi_{H F}|\underbrace{e^{-\hat{\tau}} \hat{H} e^{\hat{\tau}}}_{\bar{H}}| \Phi_{H F}\right\rangle=E_{J} .
$$

And projecting onto $\left\langle\Phi_{H F}\right| \hat{\tau}^{\dagger}$

$$
\left\langle\Phi_{H F}\left|\hat{\tau}^{\dagger} \bar{H}\right| \Phi_{H F}\right\rangle=E_{J}^{H F}\left\langle\Phi_{H F}\left|\hat{\tau}^{\dagger}\right| \Phi_{H F}\right\rangle,
$$

where $\left\langle\hat{\tau}^{\dagger}\right\rangle_{H F} \neq 0$ only for $\mathbf{k}=0$ terms in the momentumspace representation of $\hat{\tau}$ :

$$
\hat{\tau}=\frac{J}{M} \sum_{\mathbf{p}, \mathbf{q}, \mathbf{k}, \sigma} c_{\mathbf{p}-\mathbf{k}, \sigma}^{\dagger} c_{\mathbf{q}+\mathbf{k}, \bar{\sigma}}^{\dagger} c_{\mathbf{q}, \bar{\sigma}} c_{\mathbf{p}, \sigma} .
$$

Combining Eqs. (A1) and (A2) yields

$$
\left\langle(\hat{\tau}-\langle\hat{\tau}\rangle)^{\dagger} \bar{H}\right\rangle_{H F}=0,
$$


where the diagonal, $\mathbf{k}=0$ terms cancel. (In the language of the coupled-cluster approach an equivalent expression to Eq. (A4) is $\left\langle\hat{\tau}^{\dagger} H\right\rangle_{c}=0$, where $\langle\ldots\rangle_{c}$ denotes a cumulant expression over linked diagrams [114] only.) To optimize $J$ based on a single determinant $\left|\Phi_{H F}\right\rangle$ we need to solve Eq. (A4), which can also be seen as a projection of the eigenvalue equation $(\bar{H}-E)\left|\Phi_{H F}\right\rangle=0$ on the single basis of the correlation factor $\hat{\tau}$. The remaining contributing contractions $(\mathbf{k} \neq 0)$ of $(\mathrm{A} 4)$ of $\bar{H}$ are

$$
\begin{aligned}
& \left\langle\hat{\tau}^{\dagger} \bar{H}\right\rangle_{c}=\frac{1}{M^{2}} \sum_{\mathbf{p q k}, \sigma} n_{\mathbf{p}, \sigma} n_{\mathbf{q}, \bar{\sigma}}\left(1-n_{\mathbf{p}-\mathbf{k}, \sigma}\right)\left(1-n_{\mathbf{q}+\mathbf{k}, \bar{\sigma}}\right) \\
& \times\{\underbrace{\omega_{2}(J, \mathbf{p}, \mathbf{k})}_{\text {2-body }}+2 t \frac{\cosh J-1}{M}[\underbrace{N_{\bar{\sigma}}\left(\epsilon_{\mathbf{p}}+\epsilon_{\mathbf{p}-\mathbf{k}}\right)}_{\text {3-body RPA }} \\
& -\underbrace{\sum_{\mathbf{s}}\left(\epsilon_{\mathbf{p}+\mathbf{q}-\mathbf{s}}+\epsilon_{\mathbf{p}-\mathbf{q}-\mathbf{k}+\mathbf{s}}\right) n_{\mathbf{s}, \bar{\sigma}}}_{\text {3-body exchange }}]\} \text {. }
\end{aligned}
$$

Equation (A5) can be evaluated directly, or since $\hat{\tau}\left|\Phi_{H F}\right\rangle=$ $c_{H F}\left|\Phi_{H F}\right\rangle+\sum_{i} c_{i}\left|D_{i}\right\rangle$ corresponds to all the double excitation on top of the Hartree-Fock determinant, it is the sum of all the double excitation matrix elements with the Hartree-Fock determinant. The diagonal contribution again cancels with the $\langle\hat{\tau}\rangle$ term in Eq. (A4). The specific optimal $J$ values for the lattice sizes, fillings, and $U / t$ values used in this study are listed in Table VI.

\section{APPENDIX B: ANALYTIC OPTIMIZATION OF $J$ IN THE THERMODYNAMIC LIMIT AT HALF FILLING}

For an infinite system at half filling, we define

$$
\begin{gathered}
T_{0}(\mathbf{k})=\frac{1}{M} \sum_{\mathbf{q}} \Theta\left(\epsilon_{F}-\epsilon_{\mathbf{q}}\right) \Theta\left(\epsilon_{\mathbf{q}+\mathbf{k}}-\epsilon_{F}\right), \\
T_{1}(\mathbf{k})=\frac{1}{M} \sum_{\mathbf{p}} \Theta\left(\epsilon_{F}-\epsilon_{\mathbf{p}}\right) \Theta\left(\epsilon_{\mathbf{p}-\mathbf{k}}-\epsilon_{F}\right) \sum_{\mathbf{d}} e^{i(\mathbf{p}-\mathbf{k}) \cdot \mathbf{d}}, \\
T_{2}(\mathbf{k})=\frac{1}{M} \sum_{\mathbf{p}} \Theta\left(\epsilon_{F}-\epsilon_{\mathbf{p}}\right) \Theta\left(\epsilon_{\mathbf{p}-\mathbf{k}}-\epsilon_{F}\right) \sum_{\mathbf{d}} e^{i \mathbf{p} \cdot \mathbf{d}}
\end{gathered}
$$

The 2-body contributions of Eq. (22) can be expressed as

$$
\begin{aligned}
& \frac{U}{2} \frac{1}{M} \sum_{\mathbf{k}} T_{0}^{2}(\mathbf{k})-\frac{t}{M}\left[\left(e^{J}-1\right) \sum_{\mathbf{k}} T_{0}(\mathbf{k}) T_{1}(\mathbf{k})+\left(e^{-J}-1\right)\right. \\
& \left.\quad \times \sum_{\mathbf{k}} T_{0}(\mathbf{k}) T_{2}(\mathbf{k})\right]=0 .
\end{aligned}
$$

In the thermodynamic limit $(M \rightarrow \infty)$ the summation in the expression of the $T_{m}$ factors (B1)-(B3) becomes integrals:

$$
\frac{1}{M} \sum_{\mathbf{q}} \longrightarrow \frac{1}{(2 \pi)^{d}} \int d^{d} q
$$

For an unpolarized system at half filling, the factor $\Theta\left(\epsilon_{F}-\epsilon_{\mathbf{q}}\right)$ leads to a square region in the $k_{x}-k_{y}$ plane and $T_{m}(\mathbf{k})$ integrals can be easily calculated after a rotation of coordinates:

$$
k_{x}^{\prime}=\frac{1}{\sqrt{2}}\left(k_{x}-k_{y}\right), \quad k_{y}^{\prime}=\frac{1}{\sqrt{2}}\left(k_{x}+k_{y}\right) .
$$

With this rotation, $T_{0}$ is found to be symmetric with respect to $k_{x}^{\prime} \rightarrow-k_{x}^{\prime}$ and $k_{y}^{\prime} \rightarrow-k_{y}^{\prime}$, so it reduces to a function of $\left|k_{x}\right|$ and $\left|k_{y}\right|$ :

$$
\begin{aligned}
T_{0}(\mathbf{k})= & \frac{1}{(2 \pi)^{2}} \int \Theta\left(\epsilon_{F}-\epsilon_{\mathbf{q}}\right) \Theta\left(\epsilon_{\mathbf{q}+\mathbf{k}}-\epsilon_{F}\right) d^{2} q \\
= & \frac{1}{(2 \pi)^{2}}\left(\int_{-\pi / \sqrt{2}}^{-\pi / \sqrt{2}+\left|k_{x}^{\prime}\right|} \int_{-\pi / \sqrt{2}+\left|k_{y}^{\prime}\right|}^{\pi / \sqrt{2}} d q_{x}^{\prime} d q_{y}^{\prime}\right. \\
& \left.+\int_{-\pi / \sqrt{2}+\left|k_{x}^{\prime}\right|}^{\pi / \sqrt{2}} \int_{-\pi / \sqrt{2}}^{-\pi / \sqrt{2}+\left|k_{y}^{\prime}\right|} d q_{x}^{\prime} d q_{y}^{\prime}\right) \\
= & \frac{1}{(2 \pi)^{2}}\left[\sqrt{2} \pi\left(\left|k_{x}^{\prime}\right|+\left|k_{y}^{\prime}\right|\right)-2\left|k_{x}^{\prime} k_{y}^{\prime}\right|\right] \\
= & \frac{1}{(2 \pi)^{2}}\left[\pi\left(\left|k_{x}-k_{y}\right|+\left|k_{x}+k_{y}\right|\right)-\left|k_{x}^{2}-k_{y}^{2}\right|\right] .
\end{aligned}
$$

With the coordinate rotation (B5), the integrand of $T_{1}$ can be factorized as

$$
\begin{aligned}
\sum_{\mathbf{d}} e^{i(\mathbf{p}-\mathbf{k}) \cdot \mathbf{d}}= & \cos \left(p_{x}-k_{x}\right)+\cos \left(p_{y}-k_{y}\right) \\
= & \cos \left[\frac{1}{\sqrt{2}}\left(p_{x}^{\prime}-k_{x}^{\prime}\right)+\frac{1}{\sqrt{2}}\left(p_{y}^{\prime}-k_{y}^{\prime}\right)\right] \\
& +\cos \left[\frac{1}{\sqrt{2}}\left(p_{x}^{\prime}-k_{x}^{\prime}\right)-\frac{1}{\sqrt{2}}\left(p_{y}^{\prime}-k_{y}^{\prime}\right)\right] \\
= & 2 \cos \left[\frac{1}{\sqrt{2}}\left(p_{x}^{\prime}-k_{x}^{\prime}\right)\right] \cos \left[\frac{1}{\sqrt{2}}\left(p_{y}^{\prime}-k_{y}^{\prime}\right)\right],
\end{aligned}
$$

and $T_{1}$ can also be found as a function of $\left|k_{x}\right|$ and $\left|k_{y}\right|$ :

$$
T_{1}(\mathbf{k})=\frac{16}{(2 \pi)^{2}}\left[\cos \left(\frac{k_{x}-k_{y}}{2}\right) \cos \left(\frac{k_{x}+k_{y}}{2}\right)-1\right] .
$$

In a similar way $T_{2}$ can be calculated as

$$
\begin{aligned}
T_{2}(\mathbf{k}) & =\frac{16}{(2 \pi)^{2}}\left[1-\cos \left(\frac{k_{x}-k_{y}}{2}\right) \cos \left(\frac{k_{x}+k_{y}}{2}\right)\right] \\
& =-T_{1}(\mathbf{k}) .
\end{aligned}
$$

The exchange part of the three-body contribution in (22) to the correlation energy can be calculated as [using here again the rotation (B5) for $\mathbf{p}$ ]

$$
\begin{aligned}
\frac{1}{M^{2}} \sum_{\mathbf{p q k} \sigma}\left(n_{\mathbf{p}, \sigma} n_{\mathbf{q}+\mathbf{k}, \bar{\sigma}} n_{\mathbf{q}, \bar{\sigma}} \sum_{\mathbf{d}} \cos (\mathbf{p} \cdot \mathbf{d}) e^{i \mathbf{k} \cdot \mathbf{d}}\right) \\
=\frac{1}{M} \sum_{\mathbf{p k} \sigma}\left(n_{\mathbf{p}, \sigma}\left(\frac{1}{2}-T_{0}(\mathbf{k})\right) \sum_{\mathbf{d}} \cos (\mathbf{p} \cdot \mathbf{d}) e^{i \mathbf{k} \cdot \mathbf{d}}\right) \\
=\frac{2 M}{(2 \pi)^{4}} \iint_{-\pi / \sqrt{2}}^{\pi / \sqrt{2}} d p_{x}^{\prime} d p_{y}^{\prime} \iint_{-\pi}^{\pi} d k_{x} d k_{y} \\
\quad \times\left(\frac{1}{2}-\frac{1}{(2 \pi)^{2}}\left(\pi\left(\left|k_{x}-k_{y}\right|+\left|k_{x}+k_{y}\right|\right)-\left|k_{x}^{2}-k_{y}^{2}\right|\right)\right)
\end{aligned}
$$




$$
\begin{aligned}
& \left(\cos \left(\frac{p_{x}^{\prime}+p_{y}^{\prime}}{\sqrt{2}}\right) \cos \left(k_{x}\right)+\cos \left(\frac{p_{x}^{\prime}-p_{y}^{\prime}}{\sqrt{2}}\right) \cos \left(k_{y}\right)\right) \\
& =\frac{32 M}{\pi^{6}} .
\end{aligned}
$$

The final results are

$$
\begin{aligned}
& T_{0}(\mathbf{k})=\frac{1}{(2 \pi)^{2}}\left(\pi\left(\left|k_{x}-k_{y}\right|+\left|k_{x}+k_{y}\right|\right)-\left|k_{x}^{2}-k_{y}^{2}\right|\right), \\
& T_{1}(\mathbf{k})=\frac{16}{(2 \pi)^{2}}\left[\cos \left(\frac{k_{x}-k_{y}}{2}\right) \cos \left(\frac{k_{x}+k_{y}}{2}\right)-1\right], \\
& T_{2}(\mathbf{k})=-T_{1}(\mathbf{k}),
\end{aligned}
$$

and the summations can also be calculate as integrals:

$$
\begin{gathered}
\frac{1}{M} \sum_{\mathbf{k}} T_{0}^{2}(\mathbf{k})=\frac{5}{72}, \\
\frac{1}{M} \sum_{\mathbf{k}} T_{0}(\mathbf{k}) T_{1}(\mathbf{k})=-\frac{16+\pi^{4}}{\pi^{6}} .
\end{gathered}
$$

$J_{\text {opt }}^{\mathrm{TDL}}$ can be obtained by solving

$$
\frac{5 U}{144}+t \frac{16+\pi^{4}}{\pi^{6}}\left(e^{J}-e^{-J}\right)=0,
$$

which, for small $U / t$, can be approximated as

$$
J_{\mathrm{opt}}^{\mathrm{TDL}}=\operatorname{argsinh}\left(-\frac{5 U}{288 t} \times \frac{\pi^{6}}{16+\pi^{4}}\right) \approx-0.14717 \frac{U}{t} .
$$

At half filling Hartree-Fock energy of the original Hubbard Hamiltonian (18), with $\mathbf{k}=0$ in the two-body term,

$$
E_{H F}^{J=0}=\left\langle-t \sum_{\mathbf{k}, \sigma} \epsilon_{\mathbf{k}} n_{\mathbf{k}, \sigma}\right\rangle_{H F}+\frac{U}{2}\left\langle\frac{1}{M} \sum_{\mathbf{p}, \mathbf{q}, \sigma} n_{\mathbf{p}, \sigma} n_{\mathbf{q}, \bar{\sigma}}\right\rangle_{H F},
$$

results to

$$
E_{H F}^{J=0}=M\left(-t \frac{64}{(2 \pi)^{2}}+\frac{U}{4}\right)
$$

in the thermodynamic limit (TDL). The additional contributions arising due to the similarity transformation

$$
\begin{aligned}
E_{H F}^{J}= & \left\langle-2 t \frac{\cosh (J-1)}{M} \sum_{\mathbf{p}, \mathbf{q}, \sigma} \epsilon_{\mathbf{p}} n_{\mathbf{p}, \sigma} n_{\mathbf{q}, \bar{\sigma}}\right\rangle_{H F} \\
& -\left\langle 2 t \frac{\cosh (J-1)}{M^{2}} \sum_{\mathbf{p}, \mathbf{q}, \mathbf{k}, \sigma} \epsilon_{\mathbf{p}+k} n_{\mathbf{p}, \sigma} n_{\mathbf{q}+\mathbf{k}, \bar{\sigma}} n_{\mathbf{q}, \bar{s}}\right\rangle_{H F}
\end{aligned}
$$

can be estimated, with $\cosh (J-1) \approx J^{2}$ for small $J$ and Eq. (B13), as

$$
E_{H F}^{J} \approx-t J^{2} M\left(\frac{16}{(2 \pi)^{2}}+\frac{64}{\pi^{6}}\right) .
$$

Hence, the energy per site in the TDL for an unpolarized system at half filling is given by

$$
E_{\mathrm{opt}}^{\mathrm{TDL}}=-t \frac{64}{(2 \pi)^{2}}+\frac{U}{4}-t J^{2}\left(\frac{16}{(2 \pi)^{2}}+\frac{64}{\pi^{6}}\right) .
$$

\section{APPENDIX C: EXCITED STATES}

As discussed in Sec. IV A 1 the right eigenvectors of a non-Hermitian operator $\bar{H}\left|\Phi_{i}^{R}\right\rangle=E_{i}\left|\Phi_{i}^{R}\right\rangle$ are in general not orthogonal to each other. And hence the way excited states are obtained with the FCIQMC method [101] should in general not be applicable to excited states of a non-Hermitian operator, since they are sampled by orthogonalizing the $n$th excited state to all lower energy states $m<n$. But it turns out that we are still able to use the dynamically adapted shift energy $E_{i}^{S}$ of Eq. (33) as a valid estimator for the excited state energies. In Fig. 10 the difference from the exact energy, obtained by the projected $e_{p}$ and shift $e_{s}$ energy estimator, for the first 10 states of the 1D $6 e^{-}$in the 6-site, periodic, $U / t=4, \mathbf{k}=0$ Hubbard model with a correlation parameter $J=-0.1$ is shown. Also shown is the difference of the sum of the overlap of the $i$ th excited states to all lower-lying states $j$ with $E_{j}<E_{i}$, for the exact right eigenvectors obtained by exact diagonalization and the sampled eigenvectors within FCIQMC:

$$
\Delta O_{i}=\sum_{j}\left|\left\langle\Phi_{i}^{e x} \mid \Phi_{j}^{e x}\right\rangle-\left\langle\Phi_{i}^{q m c} \mid \Phi_{j}^{q m c}\right\rangle\right| \quad \forall j: E_{j}<E_{i} .
$$

As mentioned $\left\langle\Phi_{i}^{R} \mid \Phi_{j}^{R}\right\rangle \neq 0$ is possible for non-Hermitian operators, and is the case for states 3,4, and 5 shown in Fig. 10,

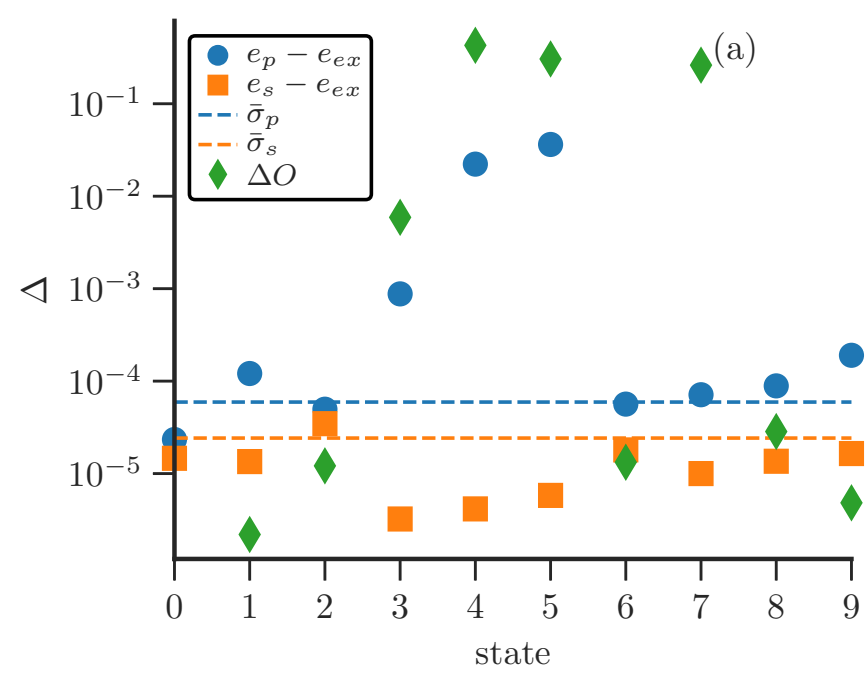

FIG. 10. Error of the first 10 eigenstate energies obtained by the projected energy $e_{p}$ and shift energy $e_{s}$ for the $6 e^{-}$in the 6-site 1D periodic Hubbard model at $U / t=4$ and $\mathbf{k}=0$ compared to exact diagonalization results. The horizontal dashed lines indicate the averaged statistical errors. The green pluses show the difference of the exact overlaps from the overlaps obtained within FCIQMC; see Eq. (C1). A correlation parameter of $J=-0.1$, initiator threshold of $n_{\text {init }}=1.2$, and maximum walker number of $N_{w}=10^{5}$ were used. (a) Exact overlap is ill defined due to degeneracy of states 6 and 7. 
indicated by a large value of $\Delta O_{i}$, since within FCIQMC the incorrect $\left\langle\Phi_{i}^{q m c} \mid \Phi_{j}^{q m c}\right\rangle \stackrel{!}{=} 0$ is tried to be enforced. The partially incorrect wave-function form is additionally marked by an large error in the projected energy $e_{p}$ compared to the exact result.

But as the $i$ th excited state is only orthogonalized to all the lower-lying excited states to converge to the next higher energy governed by the dynamics (26) and the spectrum of the Hamiltonian (1), which is unchanged by the similarity transformation (6), the shift energy remains a good energy estimator. This can clearly be seen in Fig. 10, as the shift energy is a good estimate of all the targeted eigenstates.

The only exception in Fig. 10, which could be misleading, is state number 7 , which appears to have a large error in $\Delta O_{i}$, but the projected energy is still a good estimator for the energy. This comes from the fact that states 6 and 7 are actually degenerate and thus the exact eigenvectors $\left|\Phi_{6}^{e x}\right\rangle$ and $\left|\Phi_{7}^{e x}\right\rangle$ obtained by LAPACK [115] are an arbitrary linear combination and could be chosen to both be orthogonal to the states $i<6$. The $n$th excited state in FCIQMC is obtained [101] by

$$
\left|\Phi_{n}(t+\delta t)\right\rangle=\hat{P}_{n}(t+\delta t)\left[\mathbf{1}-\delta t\left(\hat{H}-E_{n}^{S}\right)\right]\left|\Phi_{n}(t)\right\rangle
$$

with

$$
\hat{P}_{n}(t)=\mathbf{1}-\sum_{m<n} \frac{\left|\Phi_{m}(t)\right\rangle\left\langle\Phi_{m}(t)\right|}{\left\langle\Phi_{m}(t) \mid \Phi_{m}(t)\right\rangle}, \quad E_{m}<E_{n},
$$

being the Gram-Schmidt projector, which removes all contributions of lower-lying states $\left|\Phi_{m}\right\rangle$ and thus orthogonalizes $\left|\Phi_{n}\right\rangle$ to each state with $E_{m}<E_{n}$. For the set of right eigenvectors of a non-Hermitian Hamiltonian the assumption of them being orthogonal to each other does not hold in general. So this method of obtaining the excited states of $\bar{H}$ should in principle not work. But the results above indicate that the shift energy still provides a correct energy estimate.

To see why the shift energy is a valid estimate for the exact excited states energy, let us look at the right eigenvalue equation for a general (Hermitian or non-Hermitian) Hamiltonian $\hat{H}$ for the $i$ th excited state

$$
\hat{H}\left|\Psi_{i}\right\rangle=E_{i}\left|\Psi_{i}\right\rangle,
$$

where $\left|\Psi_{i}\right\rangle$ is the $i$ th right eigenvector of $\hat{H}$. We now want to show that there exists a vector $\left|\Phi_{i}\right\rangle$, which is a eigenvector of the composite operator $\hat{P}_{i} \hat{H}$ with the same eigenvalue $E_{i}$,

$$
\hat{P}_{i} \hat{H}\left|\Phi_{i}\right\rangle=E_{i}\left|\Phi_{i}\right\rangle,
$$

where $\hat{P}_{i}$ is the Gram-Schmidt projector (C3) and $\left|\Phi_{0}\right\rangle=$ $\left|\Psi_{0}\right\rangle$, which creates an orthonormal basis out of the linearindependent but not necessarily orthonormal set $\left\{\left|\Psi_{i}\right\rangle\right\}$. We assume all states to be normalized. Multiplying Eq. (C4) with $\hat{P}_{i}$ from the left, we obtain

$$
\hat{P}_{i} \hat{H}\left|\Psi_{i}\right\rangle=E_{i} \hat{P}_{i}\left|\Psi_{i}\right\rangle=E_{i}\left|\Phi_{i}\right\rangle \rightarrow\left|\Phi_{i}\right\rangle=\hat{P}_{i}\left|\Psi_{i}\right\rangle
$$

And we assume $\left|\Phi_{i}\right\rangle$ to be the desired eigenvector of $\hat{P}_{i} \hat{H}$. To show that we plug (C6) into Eq. (C5),

$$
\begin{aligned}
\hat{P}_{i} \hat{H}\left|\Phi_{i}\right\rangle & =\hat{P}_{i} \hat{H} \hat{P}_{i}\left|\Psi_{i}\right\rangle=\hat{P}_{i} \hat{H}\left(\left|\Psi_{i}\right\rangle-\sum_{j<i}\left\langle\Phi_{j} \mid \Psi_{i}\right\rangle\left|\Phi_{j}\right\rangle\right) \\
& =E_{i} \hat{P}_{i}\left|\Psi_{i}\right\rangle-\sum_{j<i} b_{j i} \hat{P}_{i} \hat{H}\left|\Phi_{j}\right\rangle \\
& =E_{i}\left|\Phi_{i}\right\rangle-\sum_{j<i} b_{j i} \hat{P}_{i} \hat{H}\left|\Phi_{j}\right\rangle
\end{aligned}
$$

with $b_{i j}=\left\langle\Phi_{j} \mid \Psi_{i}\right\rangle$. We can express $\left|\Phi_{j}\right\rangle$ in Eq. (C7) and all subsequent appearances of $\left|\Phi_{k}\right\rangle$ with $k<i$ as $\left|\Phi_{k}\right\rangle=\hat{P}_{k}\left|\Psi_{k}\right\rangle$ until we reach $\left|\Psi_{0}\right\rangle$. So the remaining thing to show is that $\hat{P}_{i}\left|\Psi_{j}\right\rangle=0$ for $i>j$.

$$
\text { For } i>j
$$

$$
\hat{P}_{i}\left|\Phi_{j}\right\rangle=\left|\Phi_{j}\right\rangle-\sum_{k<i} \underbrace{\left\langle\Phi_{k} \mid \Phi_{j}\right\rangle}_{\delta_{j k}}\left|\Phi_{k}\right\rangle=0, \quad \text { with } \quad i>j,
$$

is easy to show since $\left\{\left|\Phi_{j}\right\rangle\right\}$ is an orthonormal basis. We prove $\hat{P}_{i}\left|\Psi_{j}\right\rangle=0, j<i$ by induction. For $i=1$ we have

$$
\hat{P}_{1}\left|\Psi_{0}\right\rangle=\left|\Psi_{0}\right\rangle-\left\langle\Psi_{0} \mid \Psi_{0}\right\rangle\left|\Psi_{0}\right\rangle=0 .
$$

Let us assume $\hat{P}_{i}\left|\Psi_{j}\right\rangle=0$ for $i<j$; performing the induction step $i \rightarrow i+1$ yields

$$
\begin{aligned}
& \underline{j<i}: \hat{P}_{i+1}\left|\Psi_{j}\right\rangle=\underbrace{\hat{P}_{i}\left|\Psi_{j}\right\rangle}_{=0}-\left\langle\Phi_{i} \mid \Psi_{j}\right\rangle\left|\Phi_{i}\right\rangle \\
& =-\left\langle\Psi_{i}|\underbrace{\hat{P}_{i}^{\dagger}}_{=\hat{P}_{i}}| \Psi_{j}\right\rangle\left|\Phi_{i}\right\rangle=0, \\
& \underline{j=i}: \hat{P}_{i+1}\left|\Psi_{i}\right\rangle=\underbrace{\hat{P}_{i}\left|\Psi_{i}\right\rangle}_{=\left|\Phi_{i}\right\rangle}-\left\langle\Phi_{i} \mid \Psi_{i}\right\rangle\left|\Phi_{i}\right\rangle \\
& =\left|\Phi_{i}\right\rangle-\left\langle\Psi_{i}|\underbrace{\hat{P}_{i}}_{=\hat{P}_{i}^{2}}| \Psi_{i}\right\rangle\left|\Phi_{i}\right\rangle \\
& =\left|\Phi_{i}\right\rangle-\left\langle\Phi_{i} \mid \Phi_{i}\right\rangle\left|\Phi_{i}\right\rangle=0,
\end{aligned}
$$

where we used the Hermiticity $\hat{P}_{i}^{\dagger}=\hat{P}_{i}$ and idempotency $\hat{P}_{i}^{2}=\hat{P}_{i}$ of the projection operator. With $\hat{P}_{i}\left|\Psi_{j}\right\rangle=0$ Eq. (C7) gives the desired

$$
\hat{P}_{i} \hat{H}\left|\Phi_{i}\right\rangle=E_{i}\left|\Phi_{i}\right\rangle
$$

And this eigenvector $\left|\Phi_{i}\right\rangle$ of the composite operator $\hat{P}_{i} \hat{H}$ is the stationary vector we sample in FCIQMC. Since it has the same eigenvalue $E_{i}$, we obtain the correct excited-state energy estimate from the shift energy $E_{i}^{S}$ in the propagator (C2). The same argument holds for the long-time limit of the projection

$$
\hat{Q}_{i}\left(E_{i}^{S}\right)\left|\Psi_{i}\right\rangle=\left[\mathbf{1}-\Delta t\left(\hat{H}-E_{i}^{S}\right)\right]\left|\Psi_{i}\right\rangle=\left|\Psi_{i}\right\rangle,
$$

with stationary $\left|\Psi_{i}\right\rangle$ for $E_{i}^{S}=E_{i}$. There is an eigenvector $\left|\Phi_{i}\right\rangle$ of the composite operator

$$
\hat{P}_{i} \hat{Q}_{i}\left(E_{i}^{S}\right)\left|\Phi_{i}\right\rangle=\left|\Phi_{i}\right\rangle
$$


for $E_{i}^{S}=E_{i}$ with

$$
\left|\Phi_{i}\right\rangle=P_{i}\left|\Psi_{i}\right\rangle, \quad \text { since } \quad \hat{P}_{i} \hat{Q}_{i}\left(E_{i}^{S}\right)\left|\Psi_{i}\right\rangle=\hat{P}_{i}\left|\Psi_{i}\right\rangle .
$$

This $\left|\Phi_{i}\right\rangle$ is sampled by the walkers in a FCIQMC simulation and the shift energy $E_{i}^{S}(t)$ is adapted to keep the walker population fixed. The projected energy is in general not a good energy estimate, since

$$
\begin{aligned}
E_{i}^{P} & =\frac{\left\langle D_{i}|\hat{H}| \Phi_{i}\right\rangle}{\left\langle D_{i} \mid \Phi_{i}\right\rangle}=\frac{\left\langle D_{I}\left|\hat{H} \hat{P}_{i}\right| \Psi_{i}\right\rangle}{\left\langle D_{I}\left|\hat{P}_{i}\right| \Psi_{i}\right\rangle} \\
& =\frac{\left\langle D_{I}\left|\hat{H}\left(\mathbf{1}-\sum_{j<i}\left|\Phi_{j}\right\rangle\left\langle\Phi_{j}\right|\right)\right| \Psi_{i}\right\rangle}{\left\langle D_{I} \mid \Psi_{i}\right\rangle-\sum_{j<i}\left\langle D_{I} \mid \Phi_{j}\right\rangle\left\langle\Phi_{j} \mid \Psi_{i}\right\rangle} \\
& =\frac{E_{i} c_{I, i}-\sum_{j<i} b_{i j}\left\langle D_{I}|\hat{H}| \Phi_{j}\right\rangle}{c_{I, i}-\sum_{j<i} d_{I, j} b_{i j}} \\
& =\frac{E_{i} c_{I, i}-\sum_{j<i} b_{i j} d_{I, j} E_{j}^{P}}{c_{I, i}-\sum_{j<i} d_{I, j} b_{i j}}
\end{aligned}
$$

with $\quad c_{I, i}=\left\langle D_{I} \mid \Psi_{i}\right\rangle, b_{i j}=\left\langle\Phi_{j} \mid \Psi_{i}\right\rangle, d_{I, j}=\left\langle D_{I} \mid \Phi_{j}\right\rangle$

and $\left|D_{I}\right\rangle$ being the reference determinant of state $i$. With Eq. (C16) and knowledge of the exact eigenfunctions $\left\{\left|\Psi_{i}\right\rangle\right\}$ the excited-state energy could be calculated as

$$
E_{i}=\left[E_{i}^{P}\left(c_{I, i}-\sum_{j<i} d_{I, j} b_{i j}\right)+\sum_{j<i} b_{i j} d_{I, j} E_{j}^{P}\right] c_{I, i}^{-1} .
$$

For states where $\left\langle D_{I} \mid \Phi_{i}\right\rangle \approx c_{I, i}$ and $b_{i j} \approx 0$ the projected energy remains a good estimator for the exact $E_{i}$. But especially in cases where the exact right eigenvectors are not orthogonal to all lower-lying ones, as demonstrated in Fig. 10, the projected energy should not be trusted. Another correction for the projected energy would be

$$
\begin{gathered}
E_{i}^{P}=\frac{\left\langle D_{I}|\hat{H}| \Phi_{i}\right\rangle}{\left\langle D_{I} \mid \Phi_{i}\right\rangle} \\
\left\langle D_{I} \mid \Phi_{i}\right\rangle E_{i}^{P}=\left\langle D_{I}\left|\hat{H} \hat{P}_{i}\right| \Psi_{i}\right\rangle=\left\langle D_{I} \mid \Psi_{I}\right\rangle E_{i} \\
-\sum_{j<i}\left\langle\Phi_{j} \mid \Psi_{i}\right\rangle \underbrace{\left\langle D_{I}|\hat{H}| \Phi_{j}\right\rangle}_{=\left\langle D_{I} \mid \Phi_{j}\right\rangle E_{j}^{P}} \\
\rightarrow E_{i}=\frac{\left\langle D_{I} \mid \Phi_{i}\right\rangle E_{i}^{P}+\sum_{j<i}\left\langle\Phi_{j} \mid \Psi_{i}\right\rangle\left\langle D_{I} \mid \Phi_{j}\right\rangle E_{j}^{P}}{\left\langle D_{I} \mid \Psi_{i}\right\rangle} \\
\text { with }\left\langle D_{I} \mid \Psi_{i}\right\rangle=\left\langle D_{I} \mid \Phi_{i}\right\rangle+\sum_{j<i}\left\langle\Phi_{j} \mid \Psi_{i}\right\rangle\left\langle D_{I} \mid \Phi_{j}\right\rangle \\
\rightarrow E_{i}=\frac{\left\langle D_{I} \mid \Phi_{i}\right\rangle E_{i}^{P}+\sum_{j<i}\left\langle\Phi_{j} \mid \Psi_{i}\right\rangle\left\langle D_{I} \mid \Phi_{j}\right\rangle E_{j}^{P}}{\left\langle D_{I} \mid \Phi_{i}\right\rangle+\sum_{j<i}\left\langle\Phi_{j} \mid \Psi_{i}\right\rangle\left\langle D_{I} \mid \Phi_{j}\right\rangle} .
\end{gathered}
$$

where we can estimate the overlap $\left\langle\Phi_{j} \mid \Psi_{i}\right\rangle$ from the orthogonalization procedure.

Actually for the correct projected energy one needs to calculate

$$
\bar{E}_{i}^{P}=\frac{\left\langle D_{I}\left|\hat{P}_{i} \hat{H}\right| \Phi_{i}\right\rangle}{\left\langle D_{I} \mid \Phi_{i}\right\rangle}=E_{i}, \quad \text { since } \quad \hat{P}_{i} \hat{H}\left|\Phi_{i}\right\rangle=E_{i}\left|\Phi_{i}\right\rangle .
$$

Unfortunately the numerator of Eq. (C24) takes the following form:

$$
\left\langle D_{I}\left|\hat{P}_{i} \hat{H}\right| \Phi_{i}\right\rangle=\left\langle D_{I}|\hat{H}| \Phi_{i}\right\rangle-\sum_{j<i}\left\langle D_{I} \mid \Phi_{j}\right\rangle\left\langle\Phi_{j}|\hat{H}| \Phi_{i}\right\rangle .
$$

To calculate $\left\langle\Phi_{j}|\hat{H}| \Phi_{i}\right\rangle$ we would need the transition (reduced) density matrices [t-(R)DM] between all states $j<i$, and for the similarity-transformed momentum-space Hubbard Hamiltonian even up to the 3-body t-RDM. So we have to rely on the shift energy to yield the correct excited state energy in the ST-FCIQMC method or apply the mentioned shoelace technique in Sec. V.
[1] J. Hubbard, Proc. R. Soc. London A 276, 238 (1963).

[2] M. C. Gutzwiller, Phys. Rev. Lett. 10, 159 (1963).

[3] J. Kanamori, Prog. Theor. Phys. 30, 275 (1963).

[4] F. C. Zhang and T. M. Rice, Phys. Rev. B 37, 3759 (1988).

[5] E. Dagotto, Rev. Mod. Phys. 66, 763 (1994).

[6] D. J. Scalapino, in Handbook of High-Temperature Superconductivity: Theory and Experiment, edited by J. R. Schrieffer and J. S. Brooks (Springer, New York, 2007), pp. 495-526.

[7] S. R. White and D. J. Scalapino, Phys. Rev. B 61, 6320 (2000).

[8] D. Scalapino and S. White, Found. Phys. 31, 27 (2001).

[9] S. R. White and D. J. Scalapino, Phys. Rev. Lett. 91, 136403 (2003).

[10] L. F. Tocchio, F. Becca, A. Parola, and S. Sorella, Phys. Rev. B 78, 041101 (2008).

[11] H. Yokoyama and H. Shiba, J. Phys. Soc. Jpn. 56, 1490 (1987).

[12] D. Eichenberger and D. Baeriswyl, Phys. Rev. B 76, 180504 (2007).
[13] K. Yamaji, T. Yanagisawa, T. Nakanishi, and S. Koike Physica C (Amsterdam, Neth.) 304, 225 (1998).

[14] T. Giamarchi and C. Lhuillier, Phys. Rev. B 43, 12943 (1991).

[15] F. Becca, M. Capone, and S. Sorella, Phys. Rev. B 62, 12700 (2000).

[16] A. C. Cosentini, M. Capone, L. Guidoni, and G. B. Bachelet, Phys. Rev. B 58, R14685 (1998).

[17] H. J. M. van Bemmel, D. F. B. ten Haaf, W. van Saarloos, J. M. J. van Leeuwen, and G. An, Phys. Rev. Lett. 72, 2442 (1994).

[18] S. Zhang, J. Carlson, and J. E. Gubernatis, Phys. Rev. B 55, 7464 (1997).

[19] C.-C. Chang and S. Zhang, Phys. Rev. B 78, 165101 (2008).

[20] C.-C. Chang and S. Zhang, Phys. Rev. Lett. 104, 116402 (2010).

[21] C. N. Varney, C.-R. Lee, Z. J. Bai, S. Chiesa, M. Jarrell, and R. T. Scalettar, Phys. Rev. B 80, 075116 (2009).

[22] Y. Deng, E. Kozik, N. V. Prokof'ev, and B. V. Svistunov, Europhys. Lett. 110, 57001 (2015). 
[23] M. H. Hettler, A. N. Tahvildar-Zadeh, M. Jarrell, T. Pruschke, and H. R. Krishnamurthy, Phys. Rev. B 58, R7475 (1998).

[24] T. A. Maier, G. Alvarez, M. Summers, and T. C. Schulthess, Phys. Rev. Lett. 104, 247001 (2010).

[25] K.-S. Chen, Z. Y. Meng, S.-X. Yang, T. Pruschke, J. Moreno, and M. Jarrell, Phys. Rev. B 88, 245110 (2013).

[26] M. Potthoff, M. Aichhorn, and C. Dahnken, Phys. Rev. Lett. 91, 206402 (2003).

[27] C. Dahnken, M. Aichhorn, W. Hanke, E. Arrigoni, and M. Potthoff, Phys. Rev. B 70, 245110 (2004).

[28] A. Georges, G. Kotliar, W. Krauth, and M. J. Rozenberg, Rev. Mod. Phys. 68, 13 (1996).

[29] A. I. Lichtenstein and M. I. Katsnelson, Phys. Rev. B 62, R9283 (2000).

[30] G. Kotliar, S. Y. Savrasov, G. Pálsson, and G. Biroli, Phys. Rev. Lett. 87, 186401 (2001).

[31] E. Gull, O. Parcollet, and A. J. Millis, Phys. Rev. Lett. 110, 216405 (2013).

[32] E. Gull, M. Ferrero, O. Parcollet, A. Georges, and A. J. Millis, Phys. Rev. B 82, 155101 (2010).

[33] P. Corboz, Phys. Rev. B 93, 045116 (2016).

[34] F. Verstraete, V. Murg, and J. Cirac, Adv. Phys. 57, 143 (2008).

[35] J. P. F. LeBlanc, A. E. Antipov, F. Becca, I. W. Bulik, G. K.-L. Chan, C.-M. Chung, Y. Deng, M. Ferrero, T. M. Henderson, C. A. Jiménez-Hoyos, E. Kozik, X.-W. Liu, A. J. Millis, N. V. Prokof'ev, M. Qin, G. E. Scuseria, H. Shi, B. V. Svistunov, L. F. Tocchio, I. S. Tupitsyn, S. R. White, S. Zhang, B.-X. Zheng, Z. Zhu, and E. Gull (Simons Collaboration on the Many-Electron Problem), Phys. Rev. X 5, 041041 (2015).

[36] M. Qin, H. Shi, and S. Zhang, Phys. Rev. B 94, 085103 (2016).

[37] B.-X. Zheng, C.-M. Chung, P. Corboz, G. Ehlers, M.-P. Qin, R. M. Noack, H. Shi, S. R. White, S. Zhang, and G. K.-L. Chan, Science 358, 1155 (2017).

[38] W. F. Brinkman and T. M. Rice, Phys. Rev. B 2, 4302 (1970).

[39] M. C. Gutzwiller, Phys. Rev. 137, A1726 (1965).

[40] T. Ogawa, K. Kanda, and T. Matsubara, Prog. Theor. Phys. 53, 614 (1975).

[41] D. Vollhardt, Rev. Mod. Phys. 56, 99 (1984).

[42] F. C. Zhang, C. Gros, T. M. Rice, and H. Shiba, Supercond. Sci. Technol. 1, 36 (1988).

[43] W. Metzner and D. Vollhardt, Phys. Rev. Lett. 62, 324 (1989).

[44] C. Gros, R. Joynt, and T. M. Rice, Phys. Rev. B 36, 381 (1987).

[45] P. Horsch and T. A. Kaplan, J. Phys. C 16, L1203 (1983).

[46] T. A. Kaplan, P. Horsch, and P. Fulde, Phys. Rev. Lett. 49, 889 (1982).

[47] W. Metzner and D. Vollhardt, Phys. Rev. Lett. 59, 121 (1987).

[48] F. Gebhard and D. Vollhardt, Phys. Rev. Lett. 59, 1472 (1987).

[49] R. Jastrow, Phys. Rev. 98, 1479 (1955).

[50] M. Capello, F. Becca, M. Fabrizio, S. Sorella, and E. Tosatti, Phys. Rev. Lett. 94, 026406 (2005).

[51] J. Liu, J. Schmalian, and N. Trivedi, Phys. Rev. Lett. 94, 127003 (2005).

[52] T. Watanabe, H. Yokoyama, Y. Tanaka, and J.-i. Inoue, J. Phys. Soc. Jpn. 75, 074707 (2006).

[53] Y. M. Li and N. d'Ambrumenil, J. Appl. Phys. 73, 6537 (1993).

[54] P. W. Anderson, Science 235, 1196 (1987).

[55] B. Edegger, N. Fukushima, C. Gros, and V. N. Muthukumar, Phys. Rev. B 72, 134504 (2005).
[56] P. Anderson and N. Ong, J. Phys. Chem. Solids 67, 1 (2006).

[57] H. Yokoyama and H. Shiba, J. Phys. Soc. Jpn. 57, 2482 (1988).

[58] A. Paramekanti, M. Randeria, and N. Trivedi, Phys. Rev. Lett. 87, 217002 (2001).

[59] C. Gros, Ann. Phys. 189, 53 (1989).

[60] C. Gros, Phys. Rev. B 38, 931 (1988).

[61] J. Kaczmarczyk, J. Spałek, T. Schickling, and J. Bünemann, Phys. Rev. B 88, 115127 (2013).

[62] D. Baeriswyl, Superconductivity from repulsion: Variational results for the 2D Hubbard model in the limit of weak interaction, arXiv:1809.04916.

[63] T. K. Lee and S. Feng, Phys. Rev. B 38, 11809 (1988).

[64] H.-X. Huang, Y.-Q. Li, and F.-C. Zhang, Phys. Rev. B 71, 184514 (2005).

[65] J. Bünemann, T. Schickling, and F. Gebhard, Europhys. Lett. 98, 27006 (2012).

[66] N. Lanatà, T.-H. Lee, Y.-X. Yao, and V. Dobrosavljević, Phys. Rev. B 96, 195126 (2017).

[67] M. Fabrizio, Phys. Rev. B 95, 075156 (2017).

[68] M. M. Wysokiński and M. Fabrizio, Phys. Rev. B 95, 161106 (2017).

[69] S. Boys and N. Handy, Proc. R. Soc. London A 309, 209 (1969).

[70] S. Boys and N. Handy, Proc. R. Soc. London A 310, 63 (1969).

[71] J. O. Hirschfelder, J. Chem. Phys. 39, 3145 (1963).

[72] S. Tsuneyuki, Prog. Theor. Phys. Suppl. 176, 134 (2008).

[73] J. M. Wahlen-Strothman, C. A. Jiménez-Hoyos, T. M. Henderson, and G. E. Scuseria, Phys. Rev. B 91, 041114 (2015).

[74] E. Neuscamman, H. Changlani, J. Kinder, and G. K.-L. Chan, Phys. Rev. B 84, 205132 (2011).

[75] D. Cleland, G. H. Booth, and A. Alavi, J. Chem. Phys. 132, 041103 (2010).

[76] J. Bünemann, W. Weber, and F. Gebhard, Phys. Rev. B 57, 6896 (1998).

[77] F. Gebhard and D. Vollhardt, Phys. Rev. B 38, 6911 (1988).

[78] S. Boys and N. Handy, Proc. R. Soc. London A 310, 43 (1969).

[79] T. Yanai and T. Shiozaki, J. Chem. Phys. 136, 084107 (2012).

[80] S. Sharma, T. Yanai, G. H. Booth, C. J. Umrigar, and G. K.-L. Chan, J. Chem. Phys. 140, 104112 (2014).

[81] H. Luo, J. Chem. Phys. 136, 224111 (2012).

[82] H. Luo and A. Alavi, J. Chem. Theory Comput. 14, 1403 (2018).

[83] S. Ten-no, Chem. Phys. Lett. 330, 169 (2000).

[84] S. Ten-no, Chem. Phys. Lett. 330, 175 (2000).

[85] J. M. Wahlen-Strothman and G. E. Scuseria, J. Phys.: Condens. Matter 28, 485502 (2016).

[86] G. H. Booth, A. J. W. Thom, and A. Alavi, J. Chem. Phys. 131, 054106 (2009).

[87] Y. Ohtsuka and S. Ten-no, AIP Conf. Proc. 1456, 97 (2012).

[88] G. Golub and C. V. Loan, Matrix Computations, 2nd ed. (John Hopkins University Press, Baltimore, MD, 1989).

[89] T. Yanai, Y. Kurashige, E. Neuscamman, and G. K.-L. Chan, J. Chem. Phys. 132, 024105 (2010).

[90] T. Yanai and G. K.-L. Chan, J. Chem. Phys. 127, 104107 (2007).

[91] P. Fazekas, Physica C (Amsterdam, Neth.) 153-155, 1283 (1988). 
[92] W. Dobrautz, H. Luo, P. Rios, and A. Alavi, Combining VMC and similarity-transformed FCIQMC for the real-space Hubbard model (unpublished).

[93] J. E. Hirsch, Phys. Rev. B 31, 4403 (1985).

[94] C. Gros, R. Joynt, and T. M. Rice, Z. Phys. B 68, 425 (1987).

[95] H. Shi and S. Zhang, Phys. Rev. B 88, 125132 (2013).

[96] P. L. Rios (private communication).

[97] N. Trivedi and D. M. Ceperley, Phys. Rev. B 41, 4552 (1990).

[98] G. H. Booth, A. Alavi et al. Standalone NECI codebase designed for FCIQMC and other stochastic quantum chemistry methods, https://github.com/ghb24/NECI_STABLE.

[99] O. Gunnarsson (private communication).

[100] C. D. Sherrill and H. F. Schaefer, Adv. Quantum Chem. 34, 143 (1999).

[101] N. S. Blunt, S. D. Smart, G. H. Booth, and A. Alavi, J. Chem. Phys. 143, 134117 (2015).

[102] S. R. White, D. J. Scalapino, R. L. Sugar, E. Y. Loh, J. E. Gubernatis, and R. T. Scalettar, Phys. Rev. B 40, 506 (1989).

[103] S. Sorella, S. Baroni, R. Car, and M. Parrinello, Europhys. Lett. 8, 663 (1989).
[104] S. Zhang, J. Carlson, and J. E. Gubernatis, Phys. Rev. Lett. 78, 4486 (1997).

[105] J. Carlson, J. E. Gubernatis, G. Ortiz, and S. Zhang, Phys. Rev. B 59, 12788 (1999).

[106] S. Sorella, Phys. Rev. B 84, 241110 (2011).

[107] S. Sorella (private communication).

[108] G. Carleo, F. Becca, S. Moroni, and S. Baroni, Phys. Rev. E 82, 046710 (2010).

[109] C. Overy, G. H. Booth, N. S. Blunt, J. J. Shepherd, D. Cleland, and A. Alavi, J. Chem. Phys. 141, 244117 (2014).

[110] C. Lin, F. H. Zong, and D. M. Ceperley, Phys. Rev. E 64, 016702 (2001).

[111] D. Poilblanc, Phys. Rev. B 44, 9562 (1991).

[112] S. Sorella, Phys. Rev. B 91, 241116 (2015).

[113] P. Taylor, Coupled-cluster Methods in Quantum Chemistry (Springer, Berlin, 1994).

[114] P. Fulde, Electron Correlations in Molecules and Solids, Springer Series in Solid-State Sciences (Springer, 2012).

[115] E. Anderson, Z. Bai, C. Bischof, S. Blackford, J. Demmel, J. Dongarra, J. Du Croz, A. Greenbaum, S. Hammarling, A. McKenney, and D. Sorensen, LAPACK Users' Guide, 3rd ed. (Society for Industrial and Applied Mathematics, Philadelphia, PA, 1999). 